Acta Bot. Croat. $71(1), 51-86,2012$

\title{
Southeastern-Alpine endemic Leontodon hispidus subsp. brumatii (Cichoriaceae) in the Sava valley (central Slovenia)
}

\author{
IGOR DAKSKOBLER $^{1 *}$, ANDREJ SELIŠKAR ${ }^{2}$, BRANKO VREŠ $^{2}$ \\ ${ }^{1}$ Institute of Biology, Scientific Research Centre of the Slovenian Academy of Sciences \\ and Arts, Regional unit Tolmin, Brunov drevored 13, SI-5220 Tolmin, Slovenia \\ ${ }^{2}$ Institute of Biology, Scientific Research Centre of the Slovenian Academy of Sciences \\ and Arts, Novi trg 2, SI-1000 Ljubljana, Slovenia
}

\begin{abstract}
In the spring and summer of 2010 a number of new localities of the southeastern-Alpine endemic Leontodon hispidus subsp. brumatii were found on temporarily flooded riparian rocks in the gorge of the Sava River between the village of Sava and Zidani Most (central Slovenia). The species has so far been known only in northeastern Italy and western Slovenia (the Soča valley). In order to obtain more specific information its sites were studied phytosociologically and the communities in which it grows in the Sava and the Soča valleys compared. Two new associations were described on the basis of these comparisons: Triseto argentei-Leontodontetum brumatii ass. nov. and Leontodonti brumatii-Seslerietum calcariae ass. nov. As this endemic taxon and its endemic communities are a characteristic of riparian flora and vegetation of some Slovenian mountain rivers and as its localities in the Sava valley are explicitly disjunct and the southeasternmost in the entire known distribution area, they deserve to be studied and protected.
\end{abstract}

Key words: Leontodon brumatii, phytogeography, synsystematics, Sava, Soča, Slovenia

\section{Introduction}

Leontodon hispidus L. subsp. brumatii (Schiede ex Reichenb) T. Wraber [Leontodon hispidus var. brumatii (Schiede ex Rchb.) Fiori = L. brumatii Schiede ex Rchb.] is a southeastern-Alpine endemic, known so far only in the foothills of the Julian and Carnic Alps and along some rivers in the Friuli lowland, western Slovenia and northeastern Italy (MAYer 1952, 1958, 1960; Wraber 1998, 2007; Poldini 1991, 2002, 2009; Jogan et al. 2001; ČUŠIN 2001, 2006; ČUŠIN and DAKSKOBLER 2001; DAKSKOBLER 2005). Its localities in Slovenia are on riparian rocks and river boulders in the Nadiža riverbed in the Breginjski kot, on riparian rocks along the Soča River between Bovec and Solkan (relatively often

\footnotetext{
* Corresponding author, e-mail: igor.dakskobler@zrc-sazu.si

Copyright $^{\circledR} 2012$ by Acta Botanica Croatica, the Faculty of Science, University of Zagreb. All rights reserved.
} 
only along the middle Soča between Podselo and Plave), on similar sites along the Učja River. Reliable sites are only on the Italian side of the former border crossing Učja (WRABER 1998), very likely also downstream in the Slovenian territory, along the rivers Idrija (rarely, Golo Brdo, under Kostanjevica) and the Idrijca (very rarely, a rocky eyot on Slap ob Idrijci, under the dam at the new, small hydroelectric power plant, 9848/4, leg. et det. I. Dakskobler, 30. 5. 2010, Herbarium LJS, relevé 1 in table 2).

During our inventory of the flora in the Sava valley, between the village of Zidani most and Sava, this taxon was found at several spots on riparian rocks on both sides of the river; first on 12 May 2010 on the right bank between Trbovlje and Hrastnik, downstream from the hamlet Ribnik (Doležak) - 9856/3 (leg. I. Dakskobler et B. Vreš); on the same day on the same bank of the Sava downstream from Hrastnik, in the village of Podkraj, between the farmsteads Rus and Tohar (9856/4); two days later, on 14 May 2010, on the left bank of the Sava near Hrastnik - 9856/4 (leg. A. Seliškar). The article presents the localities and sites of this endemic taxon in the Sava valley and the communities in which it grows and compares them with its sites and communities in the Soča valley. The issue of its conservation is discussed in view of the fact that its sites along the Sava are in an area, designated for the construction of new hydroelectric power plants.

\section{Materials and methods}

Flora and vegetation along the Sava and the Soča Rivers (Figs. 1, 2) were studied according to the established central-European methods (BRAUn-Blanquet 1964, EHrEnDORFER and HAMANN 1965). Floristic records and phytosociological relevés were entered into the FloVegSi database (SELIŠKAR et al. 2003). The same application was used to make the distribution map (Fig. 2). When processing the relevés we transformed the combined cover-abundance values with numerical values (1-9) according to van der MAAREL (1979). Numerical comparisons were performed with the SYN-TAX 2000 program package (PODANI 2001). The relevés were compared by means of »(unweighted) average linkage method « - UPGMA and principal coordinates analysis (PCoA). Wishart's similarity ratio was used in all the methods. The nomenclature source for the names of vascular plants is the Mala flora Slovenije (MARTINČIČ et al. 2007), MARTINČIČ (2003) for the names of mosses and THEURILLAT (2004) for the names of the syntaxa.

\section{Ecological description of the study area}

The study area with Leontodon hispidus subsp. brumatii belongs to the pre-Alpine phytogeographical region (M. WRABER 1969). In terms of landscape regions it forms a part of the Sava valley or, more widely speaking, of the Posavsko hribovje region (PERKO and OROŽEN ADAMIČ 1998). From Litija towards Zidani Most the Sava initially still runs through a relatively wide valley which narrows at the village of Sava and remains narrow with steep banks throughout its course. On the right bank of the river, up to Hrastnik, runs a regional road, and on the left bank the double track Ljubljana-Zagreb railway. The prevailing geological bedrock consists of Triassic dolomite and limestone, in some sections (in the vicinity of Litija, Podkraj pri Hrastniku) also Permian-carbon slate claystones and sandstones (BUSER 1990). The climate is moderate continental. Mean annual air temperature in 


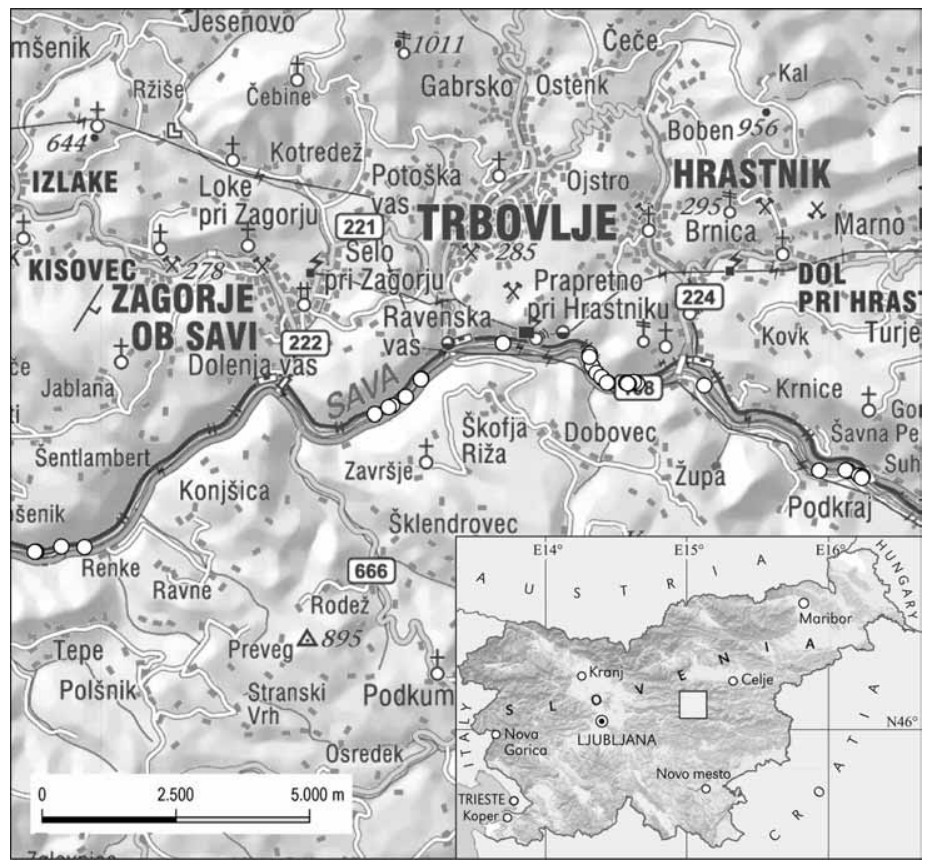

Fig. 1. Distribution of the taxon Leontodon hispidus subsp. brumatii in Slovenia with new localities in the Sava valley.

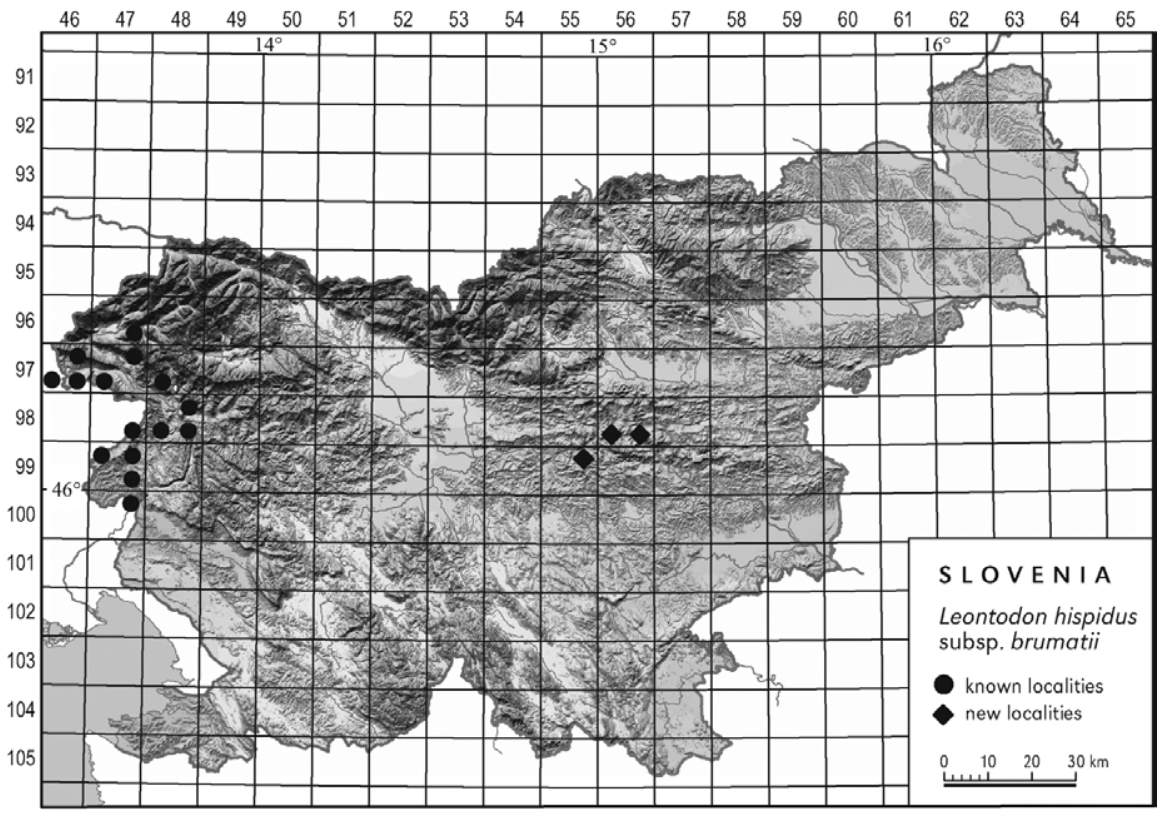

Fig. 2. Localities of the taxon Leontodon hispidus subsp. brumatii in the Sava valley (central Slovenia). 
the period $1961-1990$ was between $8{ }^{\circ} \mathrm{C}$ and $10{ }^{\circ} \mathrm{C}$, mean January temperature $-2{ }^{\circ} \mathrm{C}$ to 0 ${ }^{\circ} \mathrm{C}$ and mean July temperature $18{ }^{\circ} \mathrm{C}$ to $20{ }^{\circ} \mathrm{C}$ (CEgnAR 1998). Mean annual precipitation in the same period was between $1200 \mathrm{~mm}$ and $1400 \mathrm{~mm}$ (ZuPANČIČ 1998). The Sava River is torrential in this section and its level rapidly increases after heavy rain; rocky ledges along the river are partly or entirely flooded at least ten times a year (Fig. 3). Normally, there is a short period in May when the water level is low, and a longer period favourable for the development of the vegetation in the summer months from July to September (Hydrological data ARCHIVE 2008). Dolomite slopes above the Sava are overgrown with basophilic beech forests (Ostryo-Fagetum, Arunco-Fagetum, Hacquetio-Fagetum), and in areas where clay slates are the dominant bedrock also with acidophilous beech forests (Blechno-Fagetum). On the colluvium (hillside scree) in the gorges and at the foot of slopes there are some sites of valuable broad-leaved species (Hacquetio-Fraxinetum, Veratro nigri-Fraxinetum, Tilio cordatae-Aceretum platanoidis ostryetosum). The steepest, rockiest sites at the right bank of the Sava (Reber between the village of Sava and Mošenik; Reber between Zagorje and Trbovlje) are overgrown with basophilic forests of Scots pine and black pine (Genisto januensis-Pinetum sylvestris). On similarly steep, rocky sites on both banks of the river there are also sites of the community of pubescent oak and hop hornbeam (Querco pubescenti-Ostryetum carpinifoliae). On smaller areas on rare gravel sites grows a community of grey and red willow (Salicetum eleagno-purpureae). The banks of the Sava between Litija and Zidani Most have been changed considerably, often reinforced with dry stone walls. Towards Zidani Most the river course becomes noticeably more placid and the banks flooded, due to the dam of the Boštanj hydroelectric power plant downstream. The best preserved natural slopes with rocky ledges are in the gorge between Hrastnik and Trbovlje and it is there that most of localities of Leontodon hispidus subsp. brumatii were found.

\section{Results}

\section{Overview of the new localities of Leontodon hispidus subsp. brumatii in the Sava valley}

9856/3 (UTM 33TWM00) Slovenia, Dolenjska, the Sava valley, the right bank of the Sava downstream from the hamlet of Ribnik (Doležak), riparian rocks and pioneer grey willow stands (Salicetum eleagno-purpureae), $200 \mathrm{~m}$ a.s.l., leg. et det. I. Dakskobler et B. Vreš, 12. May 2010, Herbarium LJS; the right bank of the Sava, upstream from the hamlet of Ribnik (Doležak) and the outfall of the Ribnik stream into the Sava, 205 m a.s.l. Det. B. Vreš, 8. June 2010.

9856/3 (UTM 33T WM00) Slovenia: Štajerska, Trbovlje, the left bank of the Sava, a rocky ledge at the Trbovlje thermal power plant; also to the east and southeast of this thermal power plant, under Ringa railway tunnel and further on to the foot of the hill Vištov vrh (opposite Ribnik or Doležak), 200 m a.s.l., Det. A. Seliškar, 19. May 2010.

9856/3 (UTM 33T WM00) Slovenia: Štajerska, the Sava valley, Trbovlje, hamlet Za Savo, on several spots on the banks of the Sava under the farmsteads Vrstovšek, Frajle, Frankovič and Kos, 200 m a.s.l. Det. B. Vreš, 27. July 2010.

9856/3 (UTM 33T WM00) Slovenia: Dolenjska, the Sava valley, Mitovšek, Ribogojnica (fish hatchery), riparian rocks on the right bank of the Sava, $205 \mathrm{~m}$ a.s.1. Det. B. 
Vreš, 26. July 2010; Spodnji Šklendrovec, under the road Prusnik - Mitovšek, $210 \mathrm{~m}$ a.s.l.; Det. B. Vrě̌, 15. September 2010.

9856/4 (UTM 33T WM00) Slovenia: Dolenjska, the right bank of the Sava downstream from Hrastnik, Podkraj, at the homesteads Rus and Tohar and several spots between them (under the Ravenski hrib hill), riparian rocks, $200 \mathrm{~m}$ a.s.l., leg. et det. I. Dakskobler et B. Vreš, 12. May 2010, I. Dakskobler et B. Čušin, 17. June 2010, B. Vreš, 26. July 2010 and 7. October 2010, Herbarium LJS.

9856/4 (UTM 33T WM00) Slovenia: Štajerska, Hrastnik, the left bank of the Sava near the hamlet of $\mathrm{Za} \mathrm{Savo,} \mathrm{to} \mathrm{the} \mathrm{east} \mathrm{of} \mathrm{the} \mathrm{bridge} \mathrm{across} \mathrm{the} \mathrm{river,} \mathrm{riparian} \mathrm{rocks,} 200 \mathrm{~m}$ a.s.l., leg. et det. A. Seliškar, 14. May 2010 and 20. July 2010, Herbarium LJS.

9856/4 (UTM 33T WM00) Slovenia: Štajerska, the Sava valley, Hrastnik, the foot of the Vištov vrh hill, riparian rocks and ledges on the left bank of the Sava (under the railway track) between Trbovlje and Hrastnik, at several spots, 200 m a.s.l., leg. et det. A. Seliškar et I. Dakskobler, 18. May 2010, Herbarium LJS.

9856/4 (UTM 33T WM00) Slovenia: Dolenjska, the right bank of the Sava to the west of Hrastnik, under the filling station along the main road Zagorje-Hrastnik, $200 \mathrm{~m}$ a.s.l. Det. I. Dakskobler et A. Seliškar, 18. May 2010.

9856/4 (UTM 33TWM10) Slovenia: Stajerska, the left bank of the Sava, below the village of Suhadol, flood bank along the Sava, 195 m a.s.1. Det. A. Seliškar, 20. July 2010.

9955/2 (UTM 33TVM90) Slovenia: Dolenjska, the Sava valley, Renke, the right bank of the Sava downstream from Šuštarski most, a bridge across the Sava, 220 m a.s.l., riparian rocks., leg. et det. I. Dakskobler et B. Čušin, 17. May 2010, Herbarium LJS.

9955/2 (UTM 33TVM90) Slovenia: Štajerska, the Sava valley, Mošenik, the left bank of the Sava, to the west of the Šuštarski most (a footbridge), rocks along the Sava, at the outfall of a tufa-forming stream, $220 \mathrm{~m}$ a.s.l. Det. A. Seliškar and B. Vreš, 12. October 2010; Mošenik, the left bank of the Sava, to the west of the bridgeŠuštarski most, riparian rocks below the abandoned Mars homestead, 220 m a.s.l. Det. A. Seliškar et B. Vreš, 12. October 2010.

Leontodon brumatii was recorded on the banks of the Sava between Litija and Zidani Most at the altitude of 195 to $220 \mathrm{~m}$, at 38 microlocalities in four sections: between the villages of Sava and Zagorje (the westernmost localities are at Renke on the right and at Mošenik at the left bank of the river), between Zagorje and Trbovlje (at Mitovšek on the right and under the hamlet of Za Savo on the left bank of the river), between Trbovlje and Hrastnik (at Ribnik and the Hrastnik filling station on the right bank and at the foot of Vištov vrh on the left bank of the river, where it grows abundantly on riparian rocks) and between Hrastnik and Zidani Most or Radeče (the easternmost locality is under the village Suhadol on the left bank and at the homestead Tohar in Podkraj on the right bank) and in three quadrants - 9955/2, 9856/3 and 9856/4 (Figs. 1, 2).

\section{Phytosociological description of the sites of $L$. brumatii in the Sava valley and in the Soča valley}

Conspectus of the established syntaxa:

Asplenietea trichomanis Br.-B1. in Meier et Br.-B1. 1934 corr. Oberdorfer 1977 
Potentilletalia caulescentis Br.-B1. in Br.-B1. et Jenny 1926

Cystopteridion fragilis Richard 1972

Triseto argentei-Leontodontetum brumatii ass. nov. hoc loco

var. typica var. nov.

var. Chamaecytisus purpureus var. nov.

var. Deschampsisa cespitosa var. nov.

Festuco-Brometea Br.-Bl. et Tüxen 1943

Scorzonero-Chrysopogonetalia Horvatić et Horvat in Horvatić $1958=$ Scorzoneretalia villosae Horvatić 1975

Saturejion subspicatae Horvatić 1975

Centaurenion dichroanthae (Pignatti 1953) Poldini et Feoli Chiapella in Feoli Chiapella et Poldini 1993

Leontodonti brumatii-Seslerietum calcariae ass. nov. hoc loco

chamaecytisetum purpureae subass. nov. hoc loco

saxifragetum crustatae subass. nov. hoc loco

Salicetea purpureae Moor 1958

Salicetalia purpureae Moor 1958

Salicion eleagno-daphnoidis (Moor 1958) Grass 1993

Salicetum eleagno-purpureae Sillinger 1933 petasitetosum hybridi (Šilc et Čušin 2000) Oriolo et Poldini 2002 var. Populus nigra Dakskobler 2010

Leontodon hispidus subsp. brumatii is an endemic with a small distribution area. There are very few data on the communities in which it grows. It is known to grow on rocks (PoldinI 1991). The subspecies is not discussed on its own but together with Leontodon hispidus in the Flora alpina (AESCHIMANN et al. 2004). WRABER (1998) claims that it usually grows in a very characteristic environment, on riparian rocks that are periodically washed by river water. He stresses that the plant can also grow outside the direct influence of the water splashing, which was confirmed in our research in the Soča valley, where the studied taxon was recorded also on rocks and steep gravelly slopes that the river cannot reach even when the water level is at its highest. The specimens growing the highest above the Sava River level were found in a crevice of the retaining wall under the railway track, some $3.5 \mathrm{~m}$ above the low spring water level, and on a stony road slope approximately $5 \mathrm{~m}$ above the low spring water level. However, even these specimens are flooded when the water level is at its highest.

The rivers Soča and Idrijca are torrential and have a similar water regime to that characteristic for the Sava River (Fig. 3). Interesting is the frequent occurrence of the subspecies L. brumatii under the Doblar hydroelectric power plant where the fluctuation of the water level and in turn inundation of the subspecies' sites occurs on a daily basis. So far, no comprehensive phytosociological inventory of all its known localities in the Soča valley has been made, but we have made 15 relevés of this species at different locations over a longer time period. These relevés were compared to those from the Sava valley and thus the following results were obtained (Figs. 4, 5).

In all of the comparisons the relevés from the Sava valley (ZS) were grouped separately from the relevés from the Soča valley (PO). Two phytosociological tables were made for both regions (Tabs. 1, 2). 15 relevés made in the Sava valley on the both banks of Sava be- 
Sava - gauging station Hrastnik

Water level $(\mathrm{cm})$

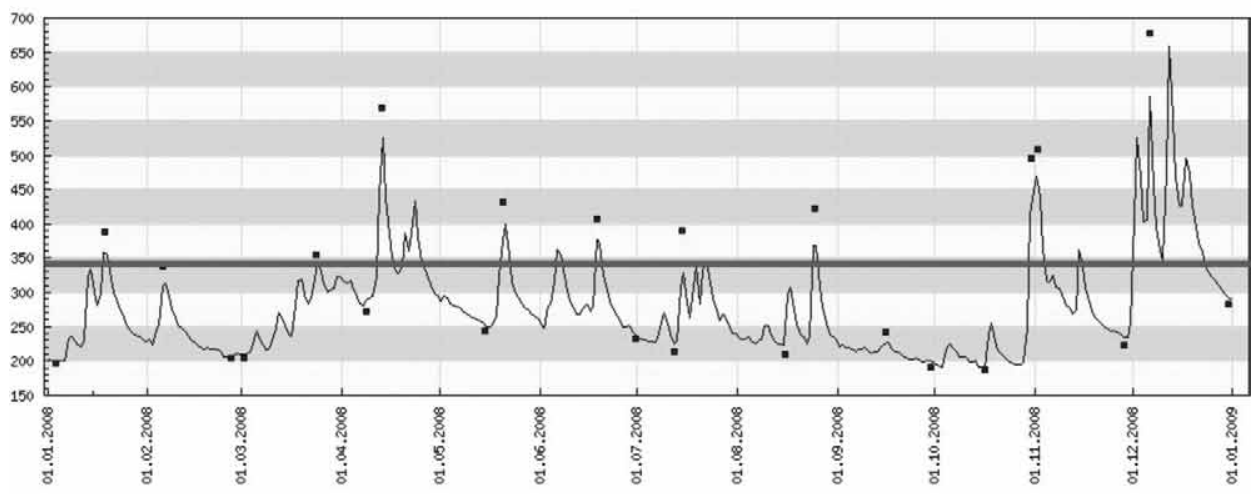

Soča gauging station Kobarid

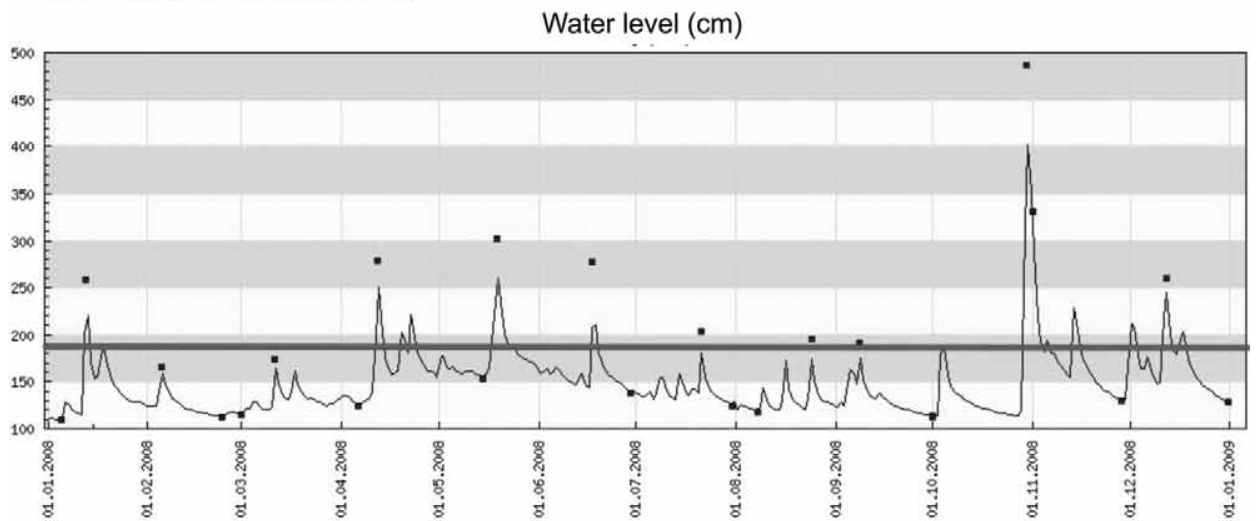

Idrijca - gauging station Hotešk

Water level $(\mathrm{cm})$

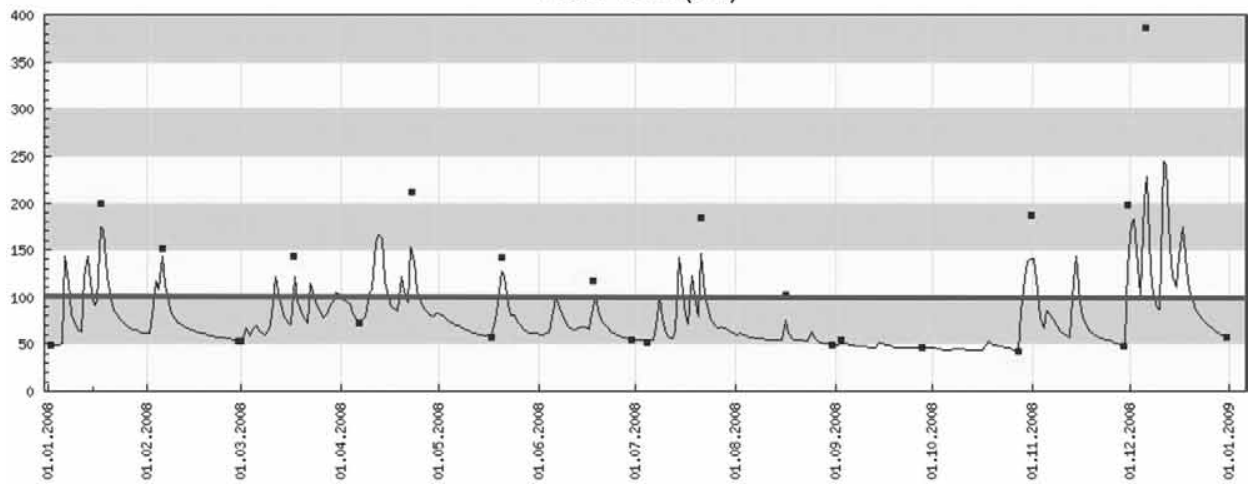

Fig. 3. Water level (in cm) of the rivers Sava, Soča and Idrijca in 2008 (according to Hydrological data archive 2008). The red line marks the threshold of flooding of the sites of Leontodon hispidus subsp. brumatii. 


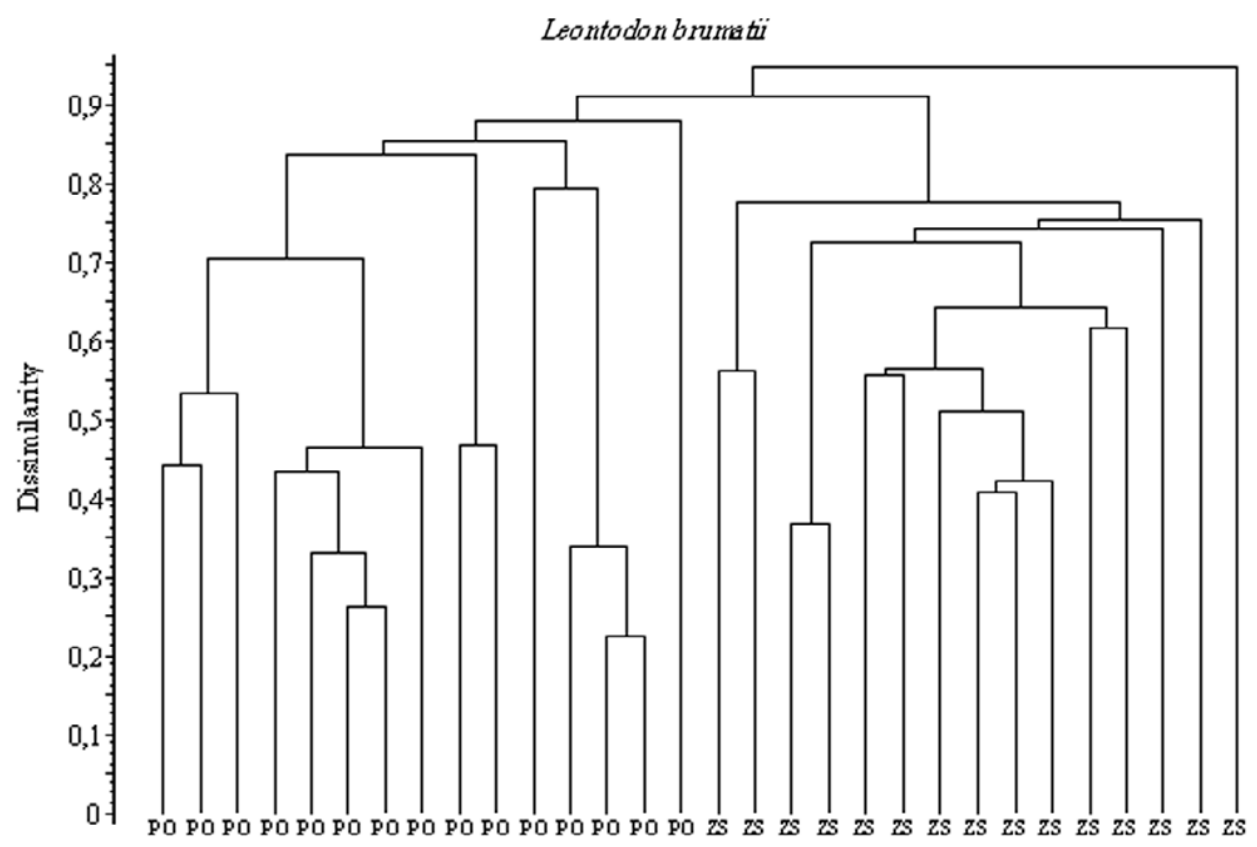

Fig. 4. Dendrogram of communities with Leontodon hispidus subsp. brumatii from the Soča valley (PO) and from the Sava valley (ZA) - UPGMA, similarity ratio.

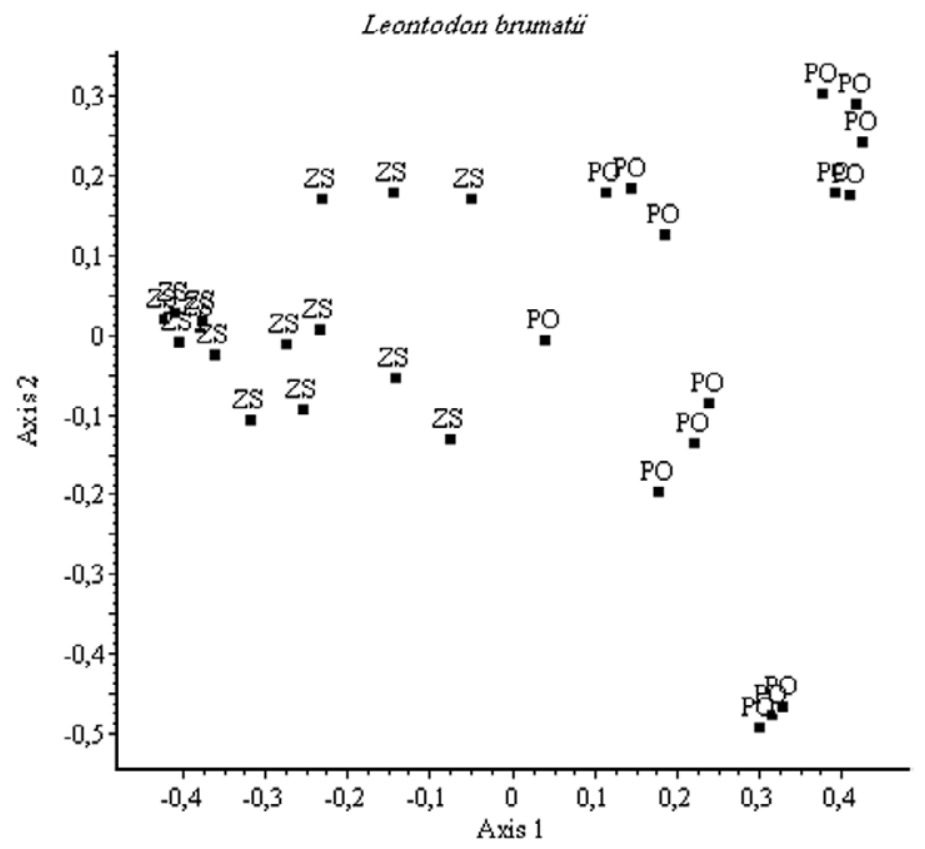

Fig. 5. Two-dimensional scatter-diagram of communities with Leontodon hispidus subsp. brumatii from the Soča valley $(\mathrm{PO})$ and from the Sava valley $(\mathrm{ZA})$ - PCoA, similarity ratio. 
Tab. 1. Communities with Leontodon hispidus subsp. brumatii in the Sava valley (central Slovenia). $\mathrm{Fl}$ - fluvisols, $\mathrm{Li}$ - lithosols.

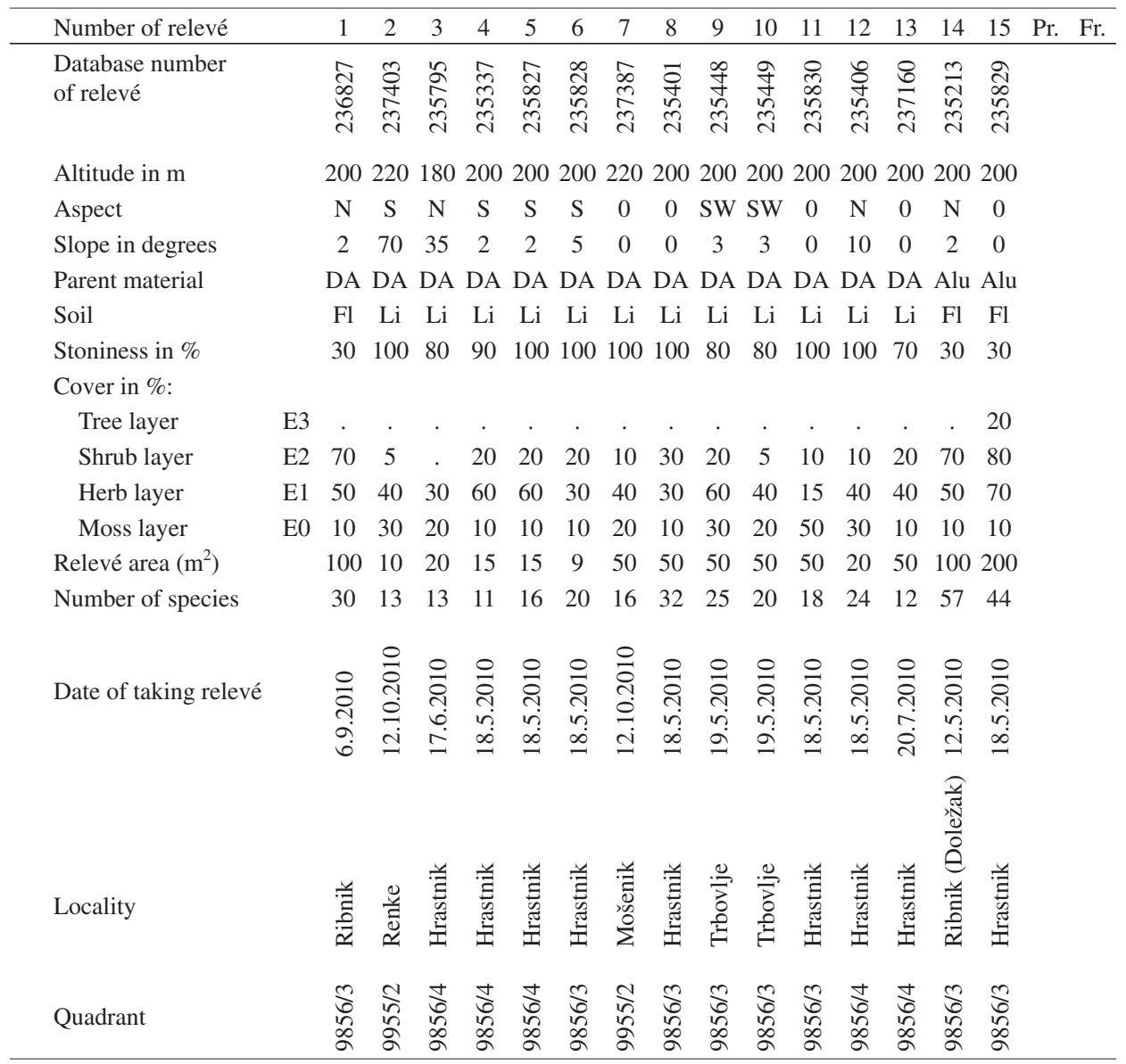

Character and differential species of the syntaxa

\begin{tabular}{|c|c|c|c|c|c|c|c|c|c|c|c|c|c|c|c|c|c|c|c|}
\hline AT & $\begin{array}{l}\text { Leontodon hispidus } \\
\text { subsp. brumatii }\end{array}$ & E1 & + & 2 & 2 & 2 & 2 & 2 & 2 & 2 & 3 & 2 & 2 & 2 & 2 & 1 & + & 15 & 100 \\
\hline M & $\begin{array}{l}\text { Brachythecium } \\
\text { rutabulum }\end{array}$ & E0 & . & 3 & 1 & 1 & 1 & + & 2 & + & 2 & 1 & 1 & 3 & 1 & + & 1 & 14 & 93 \\
\hline M & $\begin{array}{l}\text { Cinclidotus } \\
\text { fontinaloides }\end{array}$ & E0 & . & . & $\cdot$ & . & + & 1 & . & 1 & 3 & 2 & 3 & 1 & & + & & 8 & 53 \\
\hline $\mathrm{TR}$ & Trisetum argenteum & E1 & + & . & 1 & . & . & + & . & . & + & + & . & 1 & & . & . & 6 & 40 \\
\hline EP & $\begin{array}{l}\text { Chamaecytisus } \\
\text { purpureus }\end{array}$ & E1 & . & . & & 2 & 2 & 1 & . & . & . & . & . & . & & . & . & 3 & 20 \\
\hline FB & $\begin{array}{l}\text { Peucedanum } \\
\text { oreoselinum }\end{array}$ & E1 & . & . & + & 1 & 1 & . & . & . & . & . & . & . & . & . & + & 4 & 27 \\
\hline ES & $\begin{array}{l}\text { Sesleria caerulea } \\
\text { subsp. calcaria }\end{array}$ & E1 & . & + & & . & 2 & + & + & . & . & . & . & . & . & . & + & 5 & 33 \\
\hline FB & Inula ensifolia & E1 & . & . & & & . & + & & & . & & & . & & . & . & 1 & 7 \\
\hline
\end{tabular}


Tab. 1. - continued

\begin{tabular}{|c|c|c|c|c|c|c|c|c|c|c|c|c|c|c|c|c|c|c|}
\hline Number of relevé & & 1 & 2 & 3 & 4 & 5 & 6 & 7 & 8 & 9 & 10 & 11 & 12 & 13 & 14 & 15 & Pr. & Fr. \\
\hline $\begin{array}{c}\text { MA Deschampsia } \\
\text { cespitosa }\end{array}$ & E1 & 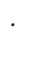 & $\cdot$ & + & . & r & 1 & 1 & 1 & 3 & 2 & 2 & 2 & 3 & 1 & 1 & 11 & 73 \\
\hline $\begin{array}{l}\text { PM Phalaris } \\
\quad \text { arundinacea }\end{array}$ & E1 & . & . & . & . & . & + & + & 1 & + & + & + & + & + & 1 & + & 10 & 67 \\
\hline SP Populus nigra & E3 & 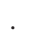 & ${ }^{\circ}$ & . & . & . & . & . & . & . & . & . & . & . & . & 2 & 1 & 7 \\
\hline SP Populus nigra & $E 2 b$ & 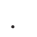 & . & . & 1 & 1 & 1 & + & 2 & 2 & + & + & + & 2 & 3 & 2 & 12 & 80 \\
\hline SP Populus nigra & $\mathrm{E} 2 \mathrm{a}$ & 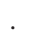 & . & . & . & . & . & . & + & . & . & 1 & . & . & 1 & 1 & 4 & 27 \\
\hline SP Salix alba & E2 & . & . & . & . & + & . & . & . & + & + & + & . & . & $\mathrm{r}$ & + & 6 & 40 \\
\hline SP Salix eleagnos & $E 2 b$ & . & . & . & . & . & + & . & . & . & . & . & 1 & . & + & 1 & 4 & 27 \\
\hline SP Salix eleagnos & E2a & . & . & . & . & . & . & . & + & . & . & . & . & . & + & + & 3 & 20 \\
\hline SP Salix purpurea & $\mathrm{E} 2 \mathrm{~b}$ & . & . & . & . & . & + & . & 1 & . & + & . & . & . & 3 & . & 4 & 27 \\
\hline SP Salix purpurea & E2a & . & + & . & . & . & . & . & . & + & . & + & . & . & 1 & . & 4 & 27 \\
\hline SP Salix purpurea & E1 & . & . & . & . & . & . & . & . & . & . & . & . & 2 & . & . & 1 & 7 \\
\hline GU Petasites hybridus & $\mathrm{E} 1$ & . & . & . & . & . & . & . & . & . & . & . & . & . & + & . & 1 & 7 \\
\hline \multicolumn{19}{|l|}{ FB Festuco-Brometea } \\
\hline Genista tinctoria & E1 & . & 2 & . & . & . & . & . & . & . & . & . & . & . & . & + & 2 & 13 \\
\hline \multicolumn{19}{|l|}{$\begin{array}{c}\text { PA Potentillion } \\
\text { anserineae }\end{array}$} \\
\hline Rorippa sylvestris & E1 & . & . & 1 & . & . & . & . & + & + & . & + & . & 1 & + & + & 7 & 47 \\
\hline Barbarea vulgaris & E1 & . & . & . & . & . & . & . & + & . & + & + & . & . & + & + & 5 & 33 \\
\hline \multicolumn{19}{|l|}{ 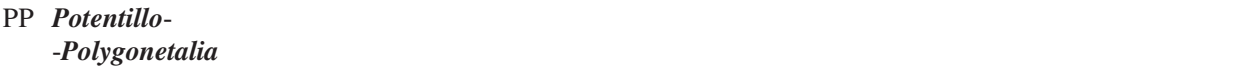 } \\
\hline Agrostis stolonifera & E1 & . & . & . & . & . & . & 1 & + & + & + & . & . & + & + & . & 6 & 40 \\
\hline Plantago major & E1 & 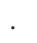 & . & . & . & . & . & . & + & + & . & + & . & . & $\mathrm{r}$ & + & 5 & 33 \\
\hline Rumex crispus & E1 & . & . & + & . & . & . & . & . & . & . & . & . & . & + & . & 2 & 13 \\
\hline \multicolumn{19}{|l|}{$\begin{array}{l}\text { MA Molinio- } \\
\text {-Arrhenatheretea }\end{array}$} \\
\hline Taraxacum officinale & E1 & . & + & . & . & . & + & . & . & + & . & . & + & . & + & + & 6 & 40 \\
\hline Trifolium pratense & E1 & . & 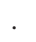 & . & . & $r^{\prime}$ & + & . & + & + & + & . & . & . & + & + & 6 & 40 \\
\hline Plantago lanceolata & E1 & . & . & . & + & + & . & . & + & . & . & . & + & . & + & . & 5 & 33 \\
\hline Achillea millefolium & E1 & . & . & . & . & . & . & . & + & . & + & . & . & . & $\mathrm{r}$ & . & 3 & 20 \\
\hline Centaurea carniolica & E1 & + & . & . & . & . & . & . & . & . & . & . & . & . & + & + & 3 & 20 \\
\hline Dactylis glomerata & E1 & . & . & . & . & . & . & . & + & . & . & . & + & . & . & + & 3 & 20 \\
\hline Galium mollugo & E1 & . & + & . & . & . & . & . & . & . & . & . & . & . & + & + & 3 & 20 \\
\hline Ranunculus acris & E1 & . & . & . & . & . & . & . & . & . & . & . & + & . & + & + & 3 & 20 \\
\hline Veronica chamaedrys & E1 & . & . & . & . & . & . & . & . & + & . & . & . & . & + & + & 3 & 20 \\
\hline Pastinaca sativa & E1 & + & $\cdot$ & $\cdot$ & . & $\cdot$ & . & . & . & . & . & . & . & . & $\mathrm{r}$ & . & 2 & 13 \\
\hline Lotus corniculatus & E1 & 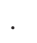 & 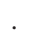 & . & . & . & + & . & . & + & . & . & . & . & . & . & 2 & 13 \\
\hline \multicolumn{19}{|l|}{ AV Artemisietea vulgaris } \\
\hline Artemisia vulgaris & E1 & + & & . & + & + & . & 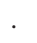 & + & + & . & . & . & . & + & + & 7 & 47 \\
\hline Erigeron аппииs & E1 & + & 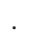 & . & . & . & $v^{\circ}$ & . & + & . & . & + & + & + & + & + & 7 & 47 \\
\hline Rumex obtusifolius & E1 & . & . & . & . & . & $\cdot$ & + & + & + & + & . & + & . & + & + & 7 & 47 \\
\hline Euphorbia esula & E1 & . & . & . & $\cdot$ & . & . & . & - & + & . & . & + & . & . & + & 3 & 20 \\
\hline Picris hieracioides & E1 & + & & . & . & & . & . & . & & . & . & . & . & . & + & 2 & 13 \\
\hline
\end{tabular}


Tab. 1. - continued

\begin{tabular}{|c|c|c|c|c|c|c|c|c|c|c|c|c|c|c|c|c|c|c|}
\hline Number of relevé & & 1 & 2 & 3 & 4 & 5 & 6 & 7 & 8 & 9 & 10 & 11 & 12 & 13 & 14 & 15 & Pr. & Fr. \\
\hline \multicolumn{19}{|l|}{$\begin{array}{l}\text { PM Phragmiti- } \\
\text {-Magnocaricetea }\end{array}$} \\
\hline Mentha aquatica & E1 & . & . & + & . & . & . & . & . & . & + & . & . & . & . & . & 2 & 13 \\
\hline \multicolumn{19}{|l|}{ SP Salicetea purpureae } \\
\hline Salix triandra & $\mathrm{E} 2 \mathrm{~b}$ & . & . & . & . & . & + & . & . & . & . & . & . & . & . & 1 & 2 & 13 \\
\hline \multicolumn{19}{|l|}{ AI Alnion incanae } \\
\hline Rubus caesius & $\mathrm{E} 2 \mathrm{a}$ & . & + & + & 1 & 1 & 1 & 1 & 1 & + & . & . & + & . & 1 & 1 & 11 & 73 \\
\hline Ulmus laevis & $\mathrm{E} 2 \mathrm{~b}$ & . & . & . & + & + & + & . & + & . & + & . & . & . & + & 1 & 7 & 47 \\
\hline Ulmus laevis & E2a & . & . & . & . & . & . & . & + & . & . & + & + & . & + & . & 4 & 27 \\
\hline $\begin{array}{l}\text { Chaerophyllum } \\
\text { hirsutum }\end{array}$ & E1 & . & . & . & . & . & . & . & . & . & . & . & . & . & + & + & 2 & 13 \\
\hline AG Alnus glutinosa & E2 & . & + & . & . & . & . & 1 & . & . & . & . & . & . & . & . & 2 & 13 \\
\hline \multicolumn{19}{|l|}{ FS Fagetalia sylvaticae } \\
\hline Fraxinus excelsior & E2a & . & . & . & . & . & . & . & + & . & . & . & . & . & + & . & 2 & 13 \\
\hline Scrophularia nodosa & E1 & . & . & . & . & . & . & . & . & . & . & . & + & . & + & . & 2 & 13 \\
\hline $\begin{array}{l}\text { Brachypodium } \\
\text { sylvaticum }\end{array}$ & E1 & . & . & . & . & . & . & . & . & . & . & . & . & . & + & + & 2 & 13 \\
\hline
\end{tabular}

\section{QF Querco-Fagetea}

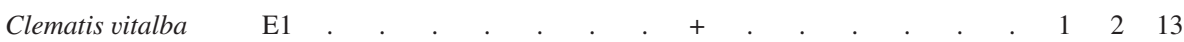

\section{RP Rhamno-Prunetea}

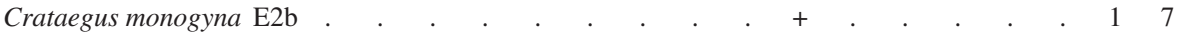

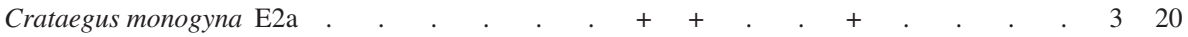

Cornus sanguinea $\quad \mathrm{E} 2 \mathrm{a}$

AR Agropyretea intermedii-repentis

Poa compressa E

E1

BT Bidentetea tripartitae

Rorippa palustris $\mathrm{E} 1+$

FC Filipendulo-

-Convolvuletea

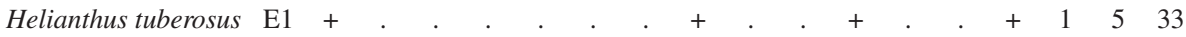

Saponaria officinalis E1 . . + + . . . . . . . . . . . . . . + +4427

Echinocystis lobata $\mathrm{E} 1+$. . . . . . . . . . . . . . . . . . . . + . 2413

Mentha longifolia $\mathrm{E} 1$. . . . . . . . . . . . . . . . . . + + 213

GU Galio-Urticetea

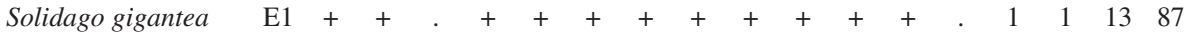

Aegopodium

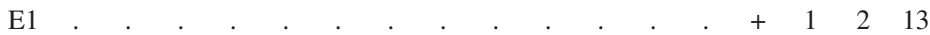
podagraria

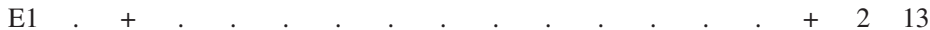

O Other species

Fallopia japonica

Poa sp.

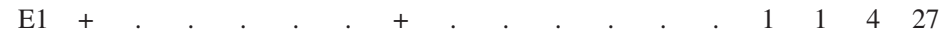

Euphorbia nutans

$\mathrm{E} 1 .+$

Malus domestica

E1

$+213$

E2a

213

M Mosses

Tortella sp.

E0

$8 \quad 53$ 


\section{Appendix to Tab. 1.}

Sporadic species: Festuco-Brometea: Petrorhagia saxifraga $+(9)$, Medicago lupulina $+(14)$, Potentillo-Polygonetalia: Ranunculus repens $+(12)$, Potentilla reptans $+(14)$, Molinio-Arrhenatheretea: Leontodon hispidus + (1), Leucanthemum ircutianum + (9), Cerastium holosteoides + (12), Crepis biennis $+(14)$, Leucanthemum vulgare $+(14)$, Rumex acetosa $+(14)$, Vicia cracca $+(14)$, Vicia sepium + (15), Artemisietea vulgaris: Cichorium intybus + (1), Tanacetum vulgare + (8), Phragmiti-Magnocaricetea: Lycopus europaeus + (8), Salicetea purpureae: Salix fragilis /E2b/ + (1), Salix fragilis /E2a/ + (2), Humulus lupulus + (1), Alnion incanae: Ulmus laevis /E1/ + (9), Peucedanum verticillare + (1), Cardamine impatiens + (14), Knautia drymeia subsp. intermedia + (14), Quercetalia pubescentis: Campanula rapunculoides + (3), Fraxinus ornus + (5), Ostrya carpinifolia + (14), Querco-Fagetea: Hieracium sabaudum + (1), Carex digitata + (2), Ficaria verna subsp. bulbifera + (14), Rhamno-Prunetea: Crataegus monogyna/E2b/+ (10), Rhamnus catharticus + (5), Rosa canina agg. + (14), Agropyretea intermedii-repentis: Equisetum arvense (14), Bidentetea tripartitae: Polygonum lapathifolium + (1), Polygonum mite + (1), Filipendulo-Convolvuletea: Calystegia sepium + (8), Lythrum salicaria + (13), Stellarietea mediae: Amaranthus retroflexus + (1), Chenopodium album + (1), Digitaria sanguinalis $+(1)$, Echinochloa crus-galli $+(1)$, Polygonum persicaria + (1), Setaria pumila + (1), Solanum nigrum + (1), Poa annиa + (10), Setaria viridis + (13), Cerastium glomeratum r (14), Other species: Acer negundo $+(1)$, Ambrosia artemisiifolia + (1), Brassica sp. + (1), Agrostis sp. + (3), Ribes rubrum + (14), Physocarpus opulifolius + (7), Mosses: Musci div. + (2).

tween Mošenik and Hrastnik were grouped together (Tab. 1). In this area, L. brumatii mainly overgrows riparian rocks, rarely also coarse, riparian gravel, both being at least periodically flooded (Figs. 6, 7). Phanerograms and some moss species grow only rarely on these rocks. In addition to L. brumatii the most abundant grass among the phanerograms is Deschampsia cespitosa. Other species, which are mostly the character species of cultivated meadows and nitrophilic ruderal communities, have only scattered and individual occurrences. Only three of the relevés in table 1 (relevés 4 to 6) slightly resemble those in the Soča valley (Tab. 2), which is mainly due to the occurrence of Chamaecytisus purpureus, Peucedanum oreoselinum and Inula ensifolia. Even the subspecies Sesleria caerulea subsp. calcaria, which usually dominates in the relevés in the Soča valley (in addition to $L$. brumatii), is relatively rare on the riparian rocks in the Sava valley and is more abundantly represented in only one relevé. Most stands with L. brumatii in the Sava valley are described as a new association Triseto argentei-Leontodontetum brumatii ass. nov. In the floristic composition as a whole there are several species which indicate a partial similarity of the studied community with the communities with dominant Phalaris arundinacea that overgrow periodically flooded sites immediately along the mid-course of rivers subject to considerable fluctuation of water level and that are classified into the alliance Phalaridion arundinaceae Kopecký 1961 and the association Rorippo-Phalaridetum Kopecký 1961 (BALÁTOVÁ-TuláčKovÁ et al. 1993). So far, this association in Slovenia has been documented with only three relevés (PETRINEC 1999). In addition to its relevés the synoptic table (Tab. 3) includes also the relevés of the same association from the border region between Slovakia and Hungary (HrivinÁK and UJHÁZY 2003). The synoptic table and numerical comparison of the three syntaxa (Figs. 8,9) clearly demonstrate that our stands cannot be classified into the association Rorippo-Phalaridetum. They comprise only a few of the species diagnostic for the class Phragmiti-Magnocaricetea Klika in Klika et Novák 1941 (Mentha aquatica, Lycopus europaeus and Phalaris arundinacea) and only P. arundinacea 
Tab. 2. Communities with Leontodon hispidus subsp. brumatii in the Soča valley (western Slovenia). $\mathrm{Li}$ - lithosols

\begin{tabular}{|c|c|c|c|c|c|c|c|c|c|c|c|c|c|c|c|c|c|}
\hline Number of relevé & & 1 & 2 & 3 & 4 & 5 & 6 & 7 & 8 & 9 & 10 & 11 & 12 & 13 & 14 & 15 & Pr. Fr. \\
\hline $\begin{array}{l}\text { Database number of } \\
\text { relevé }\end{array}$ & & 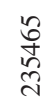 & $\begin{array}{l}\hat{\widehat{d}} \\
\hat{\tilde{d}}\end{array}$ & 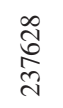 & $\begin{array}{l}\frac{n}{n} \\
\infty \\
\infty\end{array}$ & $\begin{array}{l}\frac{\nabla}{\sim} \\
\frac{\infty}{\sim}\end{array}$ & $\begin{array}{l}\frac{0}{n} \\
\frac{\infty}{\sim}\end{array}$ & $\begin{array}{l}\text { ते } \\
\text { ते }\end{array}$ & 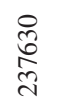 & 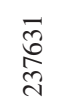 & 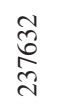 & $\begin{array}{l}m \\
\hat{b} \\
\hat{n}\end{array}$ & $\frac{\hat{\sigma}}{\hat{\sigma}}$ & 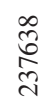 & $\begin{array}{l}\text { है } \\
\text { ते }\end{array}$ & $\begin{array}{l}\tilde{0} \\
\hat{\sigma} \\
\hat{n}\end{array}$ & \\
\hline Altitude in $\mathrm{m}$ & & 176 & 200 & 200 & 83 & 84 & 83 & 120 & 120 & 120 & 120 & 1202 & 230 & 220 & 220 & 160 & \\
\hline Aspect & & 0 & 嵒 & 空 & 学 & 学 & 3 & 㞱 & 玄 & 忞 & 忞 & 完 & 㞱 & 崩 & z & $\mathrm{z}$ & \\
\hline Slope in degrees & & 0 & 90 & 90 & 35 & 40 & 40 & 10 & 20 & 10 & 10 & 70 & 45 & 40 & 40 & 30 & \\
\hline Parent material & & A & $\mathrm{AR}$ & $\mathrm{AR}$ & $\mathrm{AL}$ & $\mathrm{AL}$ & $\mathrm{AL}$ & A & A & A & A & A & $\mathrm{AR}$ & $\mathrm{AR}$ & $\mathrm{AR}$ & $\mathrm{AR}$ & \\
\hline Soil & & $\mathrm{Li}$ & $\mathrm{Li}$ & $\mathrm{Li}$ & $\mathrm{Li}$ & $\mathrm{Li}$ & $\mathrm{Li}$ & $\mathrm{Li}$ & $\mathrm{Li}$ & $\mathrm{Li}$ & $\mathrm{Li}$ & $\mathrm{Li}$ & $\mathrm{Li}$ & $\mathrm{Li}$ & $\mathrm{Li}$ & $\mathrm{Li}$ & \\
\hline Stoniness in \% & & 100 & 100 & 100 & 100 & 100 & 100 & 90 & 100 & 90 & 60 & 100 & 30 & 30 & 30 & 90 & \\
\hline Cover in $\%$ : & & & & & & & & & & & & & & & & & \\
\hline Tree layer & E3 & 0 & 0 & 0 & 0 & 0 & 0 & 0 & 0 & 0 & 0 & 0 & 5 & 0 & 0 & 0 & \\
\hline Shrub layer & E2 & 10 & 10 & 5 & 0 & 0 & 10 & 30 & 5 & 10 & 5 & 10 & 20 & 30 & 40 & 5 & \\
\hline Herb layer & E1 & 20 & 25 & 30 & 25 & 30 & 30 & 30 & 20 & 40 & 40 & 40 & 70 & 70 & 80 & 30 & \\
\hline Moss layer & E0 & 0 & 5 & 5 & 5 & 10 & 30 & 5 & 5 & 5 & 5 & 10 & 20 & 10 & 10 & 15 & \\
\hline Relevé area $\left(\mathrm{m}^{2}\right)$ & & 50 & 50 & 50 & 10 & 10 & 10 & 10 & 10 & 50 & 10 & 15 & 100 & 100 & 100 & 15 & \\
\hline Number of species & & 14 & 29 & 18 & 20 & 13 & 18 & 25 & 22 & 25 & 22 & 36 & 37 & 37 & 33 & 17 & \\
\hline Date of taking relevé & & 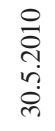 & $\frac{\widetilde{\sigma}}{\sigma}$ & $\frac{\Omega}{\sigma}$ & 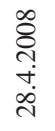 & 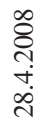 & $\begin{array}{l}\infty \\
\stackrel{0}{0} \\
\stackrel{+}{+} \\
\stackrel{\sim}{0}\end{array}$ & 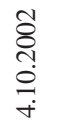 & 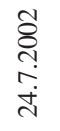 & 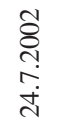 & 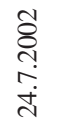 & $\begin{array}{l}\text { ¿̊ } \\
\text { ஸ̦ } \\
\stackrel{+}{+}\end{array}$ & $\begin{array}{l}\overline{8} \\
\text { तु } \\
\stackrel{0}{0}\end{array}$ & $\begin{array}{l}\stackrel{a}{\sigma} \\
\stackrel{2}{n} \\
=\end{array}$ & $\begin{array}{l}\stackrel{\partial}{\sigma} \\
\stackrel{5}{n} \\
=\end{array}$ & 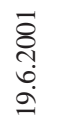 & \\
\hline Locality & & 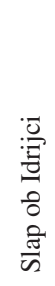 & 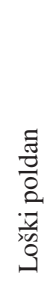 & 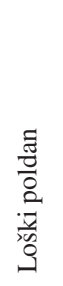 & $\begin{array}{l}\stackrel{0}{0} \\
\stackrel{0}{0} \\
\dot{z}\end{array}$ & 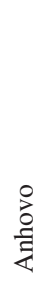 & $\begin{array}{l}\text { 家 } \\
\text { 吾 }\end{array}$ & 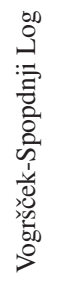 & 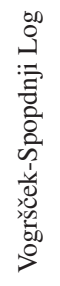 & 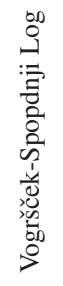 & 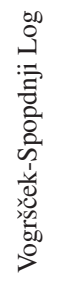 & 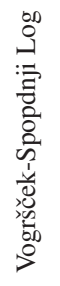 & 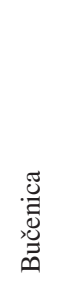 & 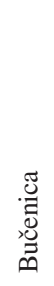 & 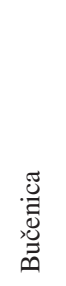 & 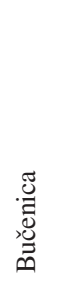 & \\
\hline Quadrant & & 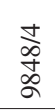 & $\begin{array}{l}\stackrel{\infty}{\infty} \\
\stackrel{+}{\circ} \\
\stackrel{\circ}{\circ}\end{array}$ & 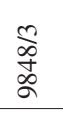 & $\begin{array}{l}\frac{1}{S} \\
\text { a }\end{array}$ & 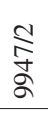 & $\frac{N}{\stackrel{f}{\sigma}}$ & $\begin{array}{l}\frac{n}{\infty} \\
\stackrel{+}{\infty} \\
\stackrel{\circ}{\infty}\end{array}$ & $\begin{array}{l}\frac{\infty}{\infty} \\
\stackrel{+}{\infty} \\
\stackrel{\alpha}{\infty}\end{array}$ & $\begin{array}{l}m \\
\infty \\
\infty \\
\infty \\
\infty\end{array}$ & $\begin{array}{l}\frac{\infty}{\infty} \\
\stackrel{+}{+} \\
\stackrel{\alpha}{\infty}\end{array}$ & $\begin{array}{l}\frac{n}{\infty} \\
\stackrel{+}{\infty} \\
\stackrel{2}{\circ}\end{array}$ & 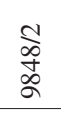 & 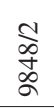 & 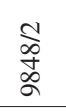 & $\begin{array}{l}1 \\
\stackrel{\infty}{\infty} \\
\stackrel{0}{\infty}\end{array}$ & \\
\hline
\end{tabular}

Character and differential species of the syntaxa

\begin{tabular}{|c|c|c|c|c|c|c|c|c|c|c|c|c|c|c|c|c|c|c|c|}
\hline ES & $\begin{array}{r}\text { Sesleria caerulea } \\
\text { subsp. calcaria }\end{array}$ & E1 & 1 & 1 & + & 2 & 2 & 2 & 2 & 2 & 2 & 2 & 2 & 3 & 3 & 3 & + & 15 & 100 \\
\hline AT & $\begin{array}{l}\text { Leontodon hispidus } \\
\text { subsp. brumatii }\end{array}$ & E1 & 1 & + & + & 2 & 2 & 1 & 1 & 1 & + & $\mathrm{r}$ & + & + & + & $\mathrm{r}$ & + & 15 & 100 \\
\hline EP & Calamagrostis varia & E1 & 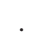 & . & . & . & . & + & + & + & + & + & 1 & + & + & + & 1 & 10 & 67 \\
\hline AT & $\begin{array}{l}\text { Hieracium } \\
\text { porrifolium }\end{array}$ & E1 & . & . & . & + & . & . & 1 & + & + & + & + & + & + & + & . & 9 & 60 \\
\hline ES & Aster bellidiastrum & E1 & & $\dot{\sigma}$ & . & . & ${ }^{\circ}$ & . & + & + & + & & 1 & + & + & + & 1 & 8 & 53 \\
\hline AT & Athamanta turbith & E1 & & 1 & 2 & . & . & + & + & 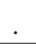 & + & . & + & . & + & + & . & 8 & 53 \\
\hline AT & Campanula carnica & E1 & & 1 & + & & & . & . & & . & . & . & . & . & & . & 2 & 13 \\
\hline AT & $\begin{array}{l}\text { Campanula } \\
\text { pyramidalis }\end{array}$ & E1 & & + & 1 & . & . & . & . & . & . & . & . & . & . & . & . & 2 & 13 \\
\hline AT & Sedum album & E1 & & + & + & r. & & & . & & . & & . & 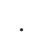 & . & . & . & 2 & 13 \\
\hline
\end{tabular}


Tab. 2. - continued

\begin{tabular}{|c|c|c|c|c|c|c|c|c|c|c|c|c|c|c|c|c|c|c|c|}
\hline & Number of relevé & & 1 & 2 & 3 & 4 & 5 & 6 & 7 & 8 & 9 & 10 & 11 & 12 & 13 & 14 & $15 \mathrm{~F}$ & Pr. 1 & Fr. \\
\hline EP & $\begin{array}{l}\text { Chamaecytisus } \\
\text { purpureus }\end{array}$ & E1 & . & . & & + & 1 & + & 1 & 2 & 2 & 2 & 2 & . & . & . & . & 8 & 53 \\
\hline FB & Carex humilis & E1 & ${ }^{\circ}$ & . & . & + & + & + & . & + & 1 & 2 & 1 & & . & . & . & 7 & 47 \\
\hline FB & $\begin{array}{l}\text { Centaurea scabiosa } \\
\text { subsp. fritschii }\end{array}$ & E1 & . & . & . & + & . & 1 & 1 & + & + & + & + & . & . & . & . & 7 & 47 \\
\hline EP & Allium ericetorum & E1 & . & . & . & + & . & . & 1 & 1 & 2 & 2 & 1 & . & . & . & . & 6 & 40 \\
\hline FB & Inula ensifolia & E1 & . & . & . & . & . & . & + & 1 & 1 & 1 & . & . & . & . & . & 42 & 27 \\
\hline EP & Erica carnea & E1 & . & . & . & . & . & . & + & . & . & . & . & 2 & 2 & 1 & + & 5 & 33 \\
\hline AT & Saxifraga crustata & E1 & . & . & . & . & . & . & . & . & . & . & . & 2 & 2 & 2 & $\mathrm{r}$ & 42 & 27 \\
\hline Mo & $\begin{array}{l}\text { Laserpitium } \\
\text { prutenicum }\end{array}$ & E1 & . & . & . & . & . & . & . & . & . & . & . & 1 & 1 & 1 & + & 42 & 27 \\
\hline TR & Petasites paradoxus & E1 & . & . & . & & . & . & . & . & . & . & . & 1 & 1 & 1 & 1 & 42 & 27 \\
\hline TR & Campanula cespitosa & E1 & . & . & . & & . & . & . & . & . & . & . & 1 & 1 & 1 & . & 3 & 20 \\
\hline ES & Rhinanthus aristatus & E1 & . & . & . & . & . & . & . & . & . & . & . & + & 1 & + & . & 32 & 20 \\
\hline FB & Stachys recta & E1 & . & . & . & . & . & . & $\cdot$ & . & . & r. & . & + & + & + & . & 32 & 20 \\
\hline \multirow[t]{3}{*}{ AT } & $\begin{array}{l}\text { Asplenietea } \\
\text { trichomanis }\end{array}$ & & & & & & & & & & & & & & & & & & \\
\hline & $\begin{array}{l}\text { Asplenium } \\
\text { ruta- muraria }\end{array}$ & E1 & . & + & + & & + & & . & . & . & & . & . & . & . & . & 3 & 20 \\
\hline & $\begin{array}{l}\text { Phyteuma } \\
\text { scheuchzeri } \\
\text { subsp. column }\end{array}$ & E1 & . & . & $\cdot$ & & . & & + & + & r & & + & & . & . & & 3 & 20 \\
\hline
\end{tabular}

\section{ES Elyno-Seslerietea}

Globularia cordifolia $\mathrm{E} 1$. . . . + . . . . . . + . . . . + . . 320

FB Festuco-Brometea

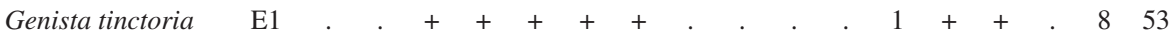

Euphorbia

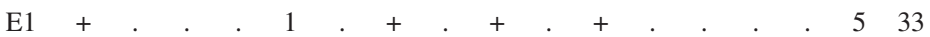

cyparissias

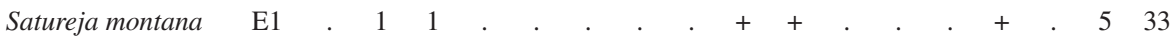
subsp. variegata

Peucedanum

E1 . . . . . . $1+++. . . \quad . \quad 533$

oreoselinum

Buphthalmum

E1 . . + . . . . . . . . 1 . 1 . $4 \quad 427$ salicifolium

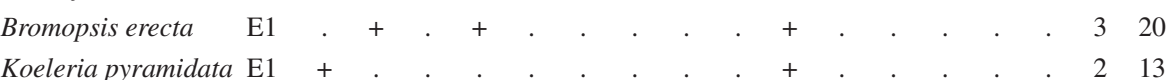

Inula hirta

$\mathrm{E} 1$. . . . . . + . . . $\mathrm{r}$. . . . 213

Ononis spinosa

Medicago falcata

E1

Gymnadenia

E1 conopsea

\section{Mo Molinion caeruleae}

SCF Parnassia palustris E

MA Molinio-

-Arrhenatheretea

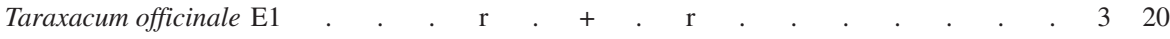

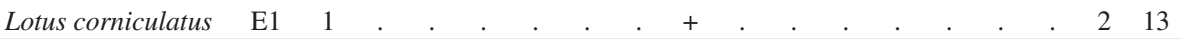


Tab. 2. - continued

\begin{tabular}{|c|c|c|c|c|c|c|c|c|c|c|c|c|c|c|c|c|c|c|c|}
\hline & Number of relevé & & 1 & 2 & 3 & 2 & 5 & 6 & 7 & 8 & 9 & 10 & 11 & 12 & 13 & 14 & 15 & Pr. & Fr. \\
\hline \multirow[t]{2}{*}{ TG } & \multicolumn{19}{|l|}{ Trifolio-Geranietea } \\
\hline & Peucedanum cervaria & $\mathrm{E} 1$ & . & + & . & . & . & + & 1 & 1 & . & 1 & + & . & . & . & . & 6 & 40 \\
\hline \multirow[t]{4}{*}{ TR } & $\begin{array}{l}\text { Thlaspietea } \\
\text { rotundifolii }\end{array}$ & & & & & & & & & & & & & & & & & & \\
\hline & $\begin{array}{l}\text { Achnatherum } \\
\text { calamagrostis }\end{array}$ & E1 & . & 1 & 1 & . & . & . & . & . & . & . & . & + & . & . & . & 3 & 20 \\
\hline & $\begin{array}{c}\text { Peucedanum } \\
\text { verticillare }\end{array}$ & E1 & . & . & . & . & . & . & . & . & . & . & $\mathrm{r}$ & + & . & . & . & 2 & 13 \\
\hline & Biscutella laevigata & E1 & . & . & . & . & . & . & . & . & . & . & . & + & 1 & . & . & 2 & 13 \\
\hline \multirow[t]{9}{*}{$\mathrm{EP}$} & Erico-Pinetea & & & & & & & & & & & & & & & & & & \\
\hline & Aster amellus & E1 & . & + & 1 & $\cdot$ & . & . & + & . & + & + & 1 & . & + & + & . & 8 & 53 \\
\hline & Leontodon incanus & E1 & . & + & + & . & . & . & + & 1 & 1 & + & 1 & . & . & . & . & 7 & 47 \\
\hline & Carex ornithopoda & E1 & . & . & . & . & . & + & . & . & $\cdot$ & . & . & + & + & + & . & 4 & 27 \\
\hline & Epipactis atrorubens & E1 & . & . & . & $\cdot$ & . & . & . & . & . & . & . & 1 & + & . & + & 3 & 20 \\
\hline & Pinus nigra & $\mathrm{E} 2 \mathrm{~b}$ & . & . & . & . & . & . & . & . & . & . & . & 1 & $\mathrm{r}$ & $\mathrm{r}$ & . & 3 & 20 \\
\hline & Pinus nigra & $\mathrm{E} 2 \mathrm{a}$ & . & . & . & $\cdot$ & . & . & . & . & . & . & . & $\mathrm{r}$ & + & . & . & 2 & 13 \\
\hline & $\begin{array}{l}\text { Polygala } \\
\text { chamaebuxus }\end{array}$ & E1 & . & . & . & . & . & . & . & . & . & . & . & 1 & 1 & 1 & . & 3 & 20 \\
\hline & $\begin{array}{l}\text { Chamaecytisus } \\
\text { hirsutus }\end{array}$ & $\mathrm{E} 2 \mathrm{a}$ & . & . & . & + & . & + & . & . & $\cdot$ & . & . & . & . & . & . & 2 & 13 \\
\hline \multicolumn{20}{|c|}{ MuA Mulgedio-Aconitetea } \\
\hline & Salix appendiculata & $\mathrm{E} 2 \mathrm{a}$ & . & . & . & 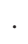 & . & . & + & . & . & . & + & + & + & + & + & 6 & 40 \\
\hline \multirow[t]{10}{*}{ QP } & $\begin{array}{l}\text { Quercetalia } \\
\text { pubescentis }\end{array}$ & & & & & & & & & & & & & & & & & & \\
\hline & Fraxinus ornus & $\mathrm{E} 2 \mathrm{~b}$ & . & . & . & . & . & . & + & . & . & . & . & . & + & 1 & . & 3 & 20 \\
\hline & Fraxinus ornus & $\mathrm{E} 2 \mathrm{a}$ & . & + & + & . & . & + & . & + & 1 & + & 1 & 1 & 1 & 1 & . & 10 & 67 \\
\hline & Fraxinus ornus & E1 & . & . & . & . & . & . & . & . & . & . & . & 1 & 1 & 1 & + & 4 & 27 \\
\hline & Ostrya carpinifolia & $\mathrm{E} 2 \mathrm{~b}$ & . & . & . & & . & . & . & . & . & . & . & 1 & 1 & 2 & . & 3 & 20 \\
\hline & Ostrya carpinifolia & $\mathrm{E} 2 \mathrm{a}$ & . & + & . & . & . & . & . & . & + & . & . & + & 1 & 1 & . & 5 & 33 \\
\hline & $\begin{array}{l}\text { Lembotropis } \\
\text { nigricans }\end{array}$ & E1 & . & . & . & $\cdot$ & . & . & . & . & . & . & + & + & + & . & . & 3 & 20 \\
\hline & Carex flacca & E1 & . & . & . & . & . & . & . & . & $\cdot$ & . & . & 1 & 1 & 1 & . & 3 & 20 \\
\hline & $\begin{array}{l}\text { Campanula } \\
\text { rapunculoides }\end{array}$ & E1 & . & . & . & . & $\mathrm{r}$ & . & . & . & . & . & + & . & . & . & . & 2 & 13 \\
\hline & Clematis recta & E1 & . & . & . & - & . & . & . & . & . & . & + & . & + & . & . & 2 & 13 \\
\hline \multirow[t]{6}{*}{ FS } & Fagetalia sylvaticae & & & & & & & & & & & & & & & & & & \\
\hline & Mycelis muralis & E1 & . & + & + & - & . & . & . & . & . & . & . & . & . & . & . & 2 & 13 \\
\hline & Anemone trifolia & E1 & . & . & . & - & . & ${ }^{\circ}$ & . & ${ }^{\circ}$ & . & . & + & . & . & . & . & 1 & 7 \\
\hline & Lathyrus vernus & E1 & . & . & . & . & . & $\cdot$ & . & . & . & . & + & . & . & . & . & 1 & 7 \\
\hline & $\begin{array}{l}\text { Ranunculus } \\
\text { lanuginosus }\end{array}$ & E1 & . & . & . & . & . & . & . & . & . & . & $\mathrm{r}$ & . & . & . & . & 1 & 7 \\
\hline & Tilia cordata & $\mathrm{E} 2 \mathrm{a}$ & + & . & . & $\cdot$ & . & . & . & . & $\cdot$ & . & $\mathrm{r}$ & . & . & . & . & 2 & 13 \\
\hline \multirow[t]{3}{*}{ QF } & Querco-Fagetea & & & & & & & & & & & & & & & & & & \\
\hline & Clematis vitalba & E2a & . & + & + & + & + & + & . & $\cdot$ & + & + & . & . & . & . & . & 7 & 47 \\
\hline & Carex digitata & E1 & . & . & . & . & . & . & . & . & . & . & . & . & + & 1 & . & 2 & 13 \\
\hline
\end{tabular}


Tab. 2. - continued

\begin{tabular}{|c|c|c|c|c|c|c|c|c|c|c|c|c|c|c|c|c|c|c|c|}
\hline & Number of relevé & & 1 & 2 & 3 & 4 & 5 & 6 & 7 & 8 & 9 & 10 & 11 & 12 & 13 & 14 & 15 & Pr. & Fr. \\
\hline \multirow[t]{4}{*}{$\overline{\mathrm{SP}}$} & Salicetea purpureae & & & & & & & & & & & & & & & & & & \\
\hline & Populus nigra & $\mathrm{E} 2 \mathrm{a}$ & + & . & . & . & + & + & . & . & . & . & . & . & . & . & . & 3 & 20 \\
\hline & Salix eleagnos & E2 & 1 & . & . & . & . & . & . & . & . & . & . & . & + & + & . & 3 & 20 \\
\hline & Salix purpurea & $\mathrm{E} 2 \mathrm{a}$ & + & . & . & . & . & + & . & . & . & . & . & . & . & . & + & 3 & 20 \\
\hline \multirow[t]{2}{*}{ AV } & Artemisietea vulgaris & & & & & & & & & & & & & & & & & & \\
\hline & Erigeron аппииs & E1 & + & . & . & $\mathrm{r}$ & . & . & . & . & . & . & . & . & . & . & . & 2 & 13 \\
\hline \multirow[t]{2}{*}{$\mathrm{O}$} & Other species & & & & & & & & & & & & & & & & & & \\
\hline & Festuca sp. & E1 & . & . & + & . & . & . & . & . & + & . & . & . & . & . & . & 2 & 13 \\
\hline \multirow[t]{10}{*}{ M } & Mosses & & & & & & & & & & & & & & & & & & \\
\hline & $\begin{array}{l}\text { Schistidium } \\
\text { apocarpum }\end{array}$ & E0 & . & 1 & . & . & . & . & 1 & + & + & 1 & + & . & . & . & . & 6 & 40 \\
\hline & Tortella sp. & E0 & . & + & . & + & 1 & . & . & . & . & . & . & 1 & 1 & 1 & . & 6 & 40 \\
\hline & Tortella tortuosa & E0 & . & . & . & . & . & . & 1 & 1 & 1 & 1 & + & . & . & . & 1 & 6 & 40 \\
\hline & $\begin{array}{l}\text { Cinclidotus } \\
\text { fontinaloides }\end{array}$ & E0 & . & . & . & + & 1 & 2 & . & . & . & . & . & . & . & . & . & 3 & 20 \\
\hline & Ctenidium molluscum & E0 & . & . & . & . & . & . & . & . & . & . & . & 1 & 1 & + & . & 3 & 20 \\
\hline & Fissidens dubius & E0 & . & . & . & . & . & . & & & & & . & + & + & + & . & 3 & 20 \\
\hline & Collema sp. & E0 & . & + & + & . & . & . & . & . & . & . & . & . & . & . & . & 2 & 13 \\
\hline & Dicranum sp. & E0 & . & . & . & . & . & . & . & . & . & . & . & + & + & . & . & 2 & 13 \\
\hline & Musci div. & E0 & . & & . & . & . & & . & . & . & . & . & + & . & . & 2 & 2 & 13 \\
\hline
\end{tabular}

Appendix to Tab. 2.

Sporadic species: Asplenietea trichomanis: Hieracium austriacum $+(1)$, Calamintha einseleana + (2), Sedum maximum + (2), Asplenium trichomanes + (2), Ceterach javorkeanum + (2), Paederota lutea + (15), Elyno-Seslerietea: Erigeron glabratus + (8), Festuco-Brometea: Allium carinatum subsp. pulchellum $+(2)$, Thymus praecox $+(4)$, Salvia pratensis $+(7)$, Trifolium montanum $+(11)$, Trifolio-Geranietea: Digitalis grandiflora $+(2)$, Verbascum lychnitis $\mathrm{r}(2)$, Vincetoxicum hirundinaria + (7), Lilium bulbiferum r (11), Thlaspietea rotundifolii: Hieracium bifidum + (1), Hieracium piloselloides + (1), Trisetum argenteum + (4), Silene vulgaris subsp. glareosa + (13), Erico-Pinetea: Molinia caerulea subsp. arundinacea + (14), Pinus sylvestris r (14), Vaccinio-Piceetea: Solidago virgaurea + (11), Picea abies + (14), Quercetalia pubescentis: Ostrya carpinifolia /E1/ 1 (12), Arabis turrita $+(2)$, Coronilla emerus subsp. emeroides r (6), Sorbus aria $+(11)$, Coronilla emerus subsp. emerus + (14), Fagetalia sylvaticae: Anemone trifolia + (11), Lathyrus vernus + (11), Ranunculus lanuginosus $\mathrm{r}$ (11), Tilia cordata /E2a/ r (11), Tilia cordata /E1/ + (12), Fagus sylvatica /E2/ r (12), Fagus sylvatica /E1/ + (12), Querco-Fagetea: Ulmus minor + (4), Pyrus pyraster + (9), Listera ovata + (11), Corylus avellana $\mathrm{r}(12)$, Quercus robur $\mathrm{r}$ (12), Salicetea purpureae: Salix eleagnos /E1/ + (12), Salix purpurea /E1/ + (4), Artemisietea vulgaris: Artemisia vulgaris + (1), Melilotus albus $\mathrm{r}(8)$, Other species: Mentha sp. + (15), Robinia pseudacacia r (5), Mosses: Homalothecium sericeum + (2), Encalypta streptocarpa $+(11)$

occurs at a higher frequency, but in modest abundance (mainly + ). The decisive factor for the synsystematic classification of the studied stands is therefore the dominant L. brumatii, which is characteristic of wet rocks. Based on the available material the most sensible classification of the new association is therefore into the alliance Cystopteridion fragilis, order Potentilletalia caulescentis and class Asplenietea trichomanis, even though no other diagnostic species of the alliance, order and class have been recorded in these stands. 


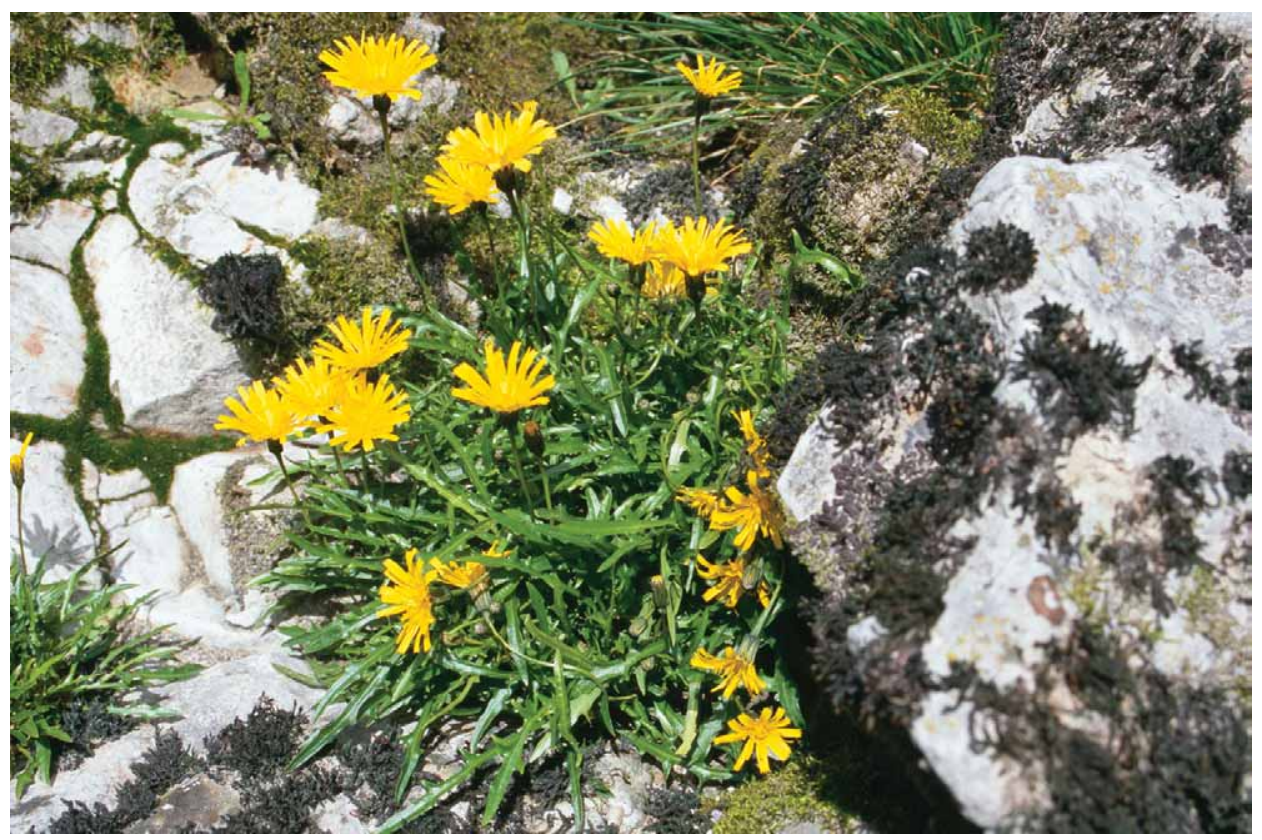

Fig. 6. Leontodon hispidus subsp. brumatii on temporarily flooded riparian rocks in the Sava valley (central Slovenia) (Photo I. Dakskobler).

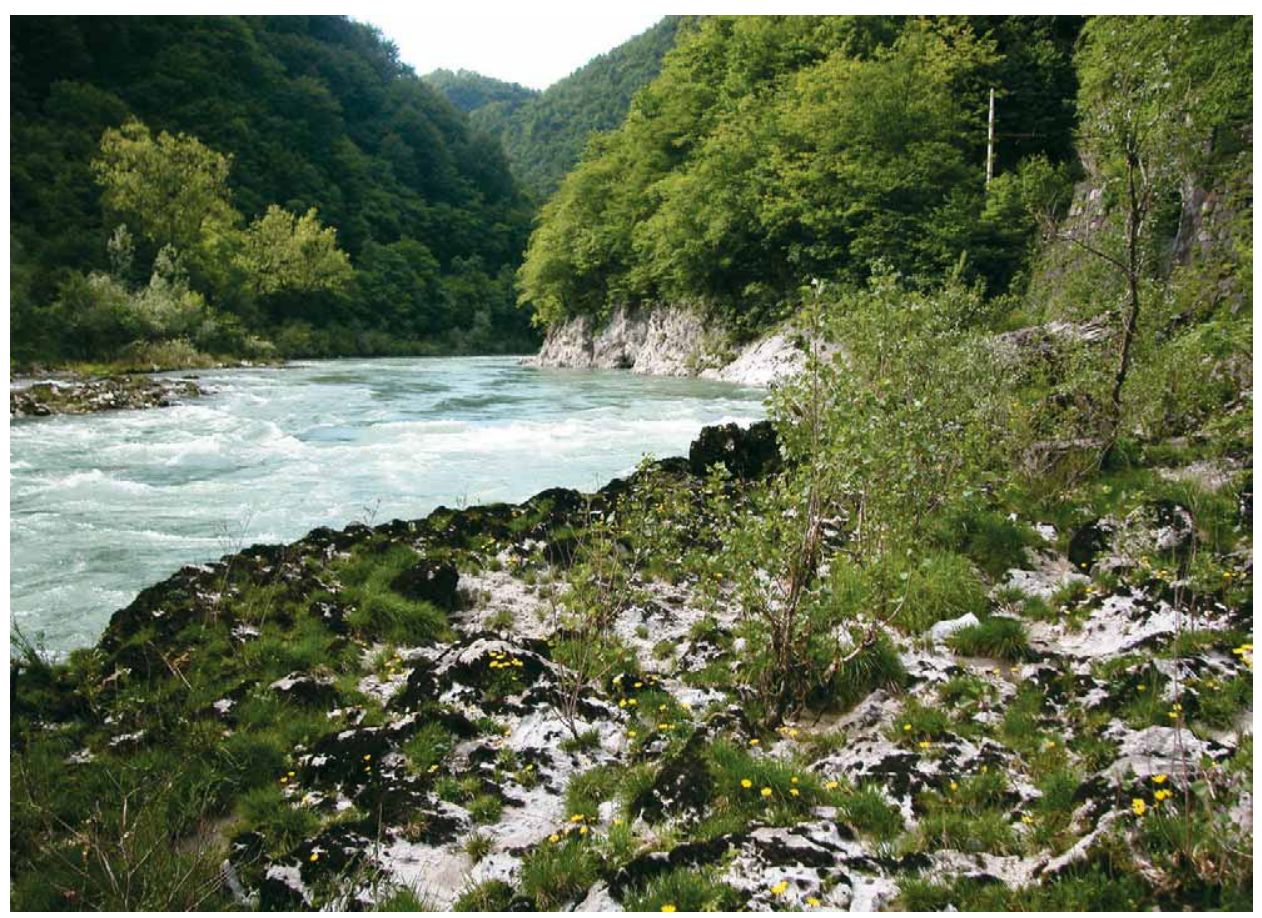

Fig. 7. Typical site (habitat) of the Triseto argentei-Leontodontetum brumatii community in the Sava valley (Photo A. Seliškar). 
Tab. 3. Synoptic table of the three riparian communities.

\begin{tabular}{|c|c|c|c|c|c|c|c|c|c|}
\hline \multicolumn{2}{|l|}{ Succesive number } & 1 & 2 & 3 & Succesive number & & 1 & 2 & 3 \\
\hline \multicolumn{2}{|l|}{ Number of relevé } & 12 & 3 & 10 & Number of relevé & & 12 & 3 & 10 \\
\hline \multicolumn{2}{|l|}{ Author } & DSV & VP & $\mathrm{HU}$ & Author & & DSV & VP & $\mathrm{HU}$ \\
\hline \multicolumn{2}{|l|}{$\underline{\text { Sign }}$} & $\mathrm{TL}$ & $\mathrm{RP}$ & RP1 & Sign & & $\mathrm{TL}$ & $\mathrm{RP}$ & RP1 \\
\hline \multicolumn{2}{|l|}{ Asplenietea trichomanis } & & & & Bidens frondosa & E1 & . & . & 20 \\
\hline $\begin{array}{l}\text { Leontodon hispidus subsp. } \\
\text { brumatii }\end{array}$ & E1 & 100 & & . & $\begin{array}{l}\text { Atriplex prostrata } \\
\text { Galio-Urticetea }\end{array}$ & E1 & . & . & 10 \\
\hline \multicolumn{2}{|l|}{ Thlaspietea rotundifolii } & & & & Solidago gigantea & E1 & 83 & . & . \\
\hline Trisetum argenteum & E1 & 42 & . & . & Urtica dioica & E1 & 8 & 33 & 90 \\
\hline \multicolumn{2}{|l|}{ Phragmiti-Magnocaricetea } & & & & Glechoma hederacea & E1 & . & . & 10 \\
\hline Phalaris arundinacea & E1 & 67 & 100 & 100 & Lamium maculatum & E1 & . & . & 10 \\
\hline Mentha aquatica & E1 & 17 & · & & Artemisietea vulgaris & & & & \\
\hline Lycopus europaeus & E1 & 8 & 100 & 10 & Rumex obtusifolius & E1 & 42 & . & 10 \\
\hline Veronica anagallis-aquatica & E1 & . & 66 & . & Artemisia vulgaris & E1 & 33 & . & $\cdot$ \\
\hline Glyceria notata & E1 & . & 66 & . & Erigeron aпnииs & E1 & 33 & . & . \\
\hline Leersia oryzoides & E1 & . & 66 & . & Euphorbia esula & E1 & 20 & . & . \\
\hline Rumex hydrolapathum & E1 & . & 66 & . & Tanacetum vulgare & E1 & 8 & . & . \\
\hline Veronica becabunga & E1 & . & 33 & . & Stellarietea mediae & & & & \\
\hline Scrophularia umbrosa & E1 & . & 33 & . & Роа аппиа & E1 & 8 & . & . \\
\hline Berula erecta & E1 & . & 33 & . & Setaria viridis & E1 & 8 & . & . \\
\hline Carex pseudocyperus & E1 & . & 33 & . & Polgonum persicaria & E1 & . & 33 & . \\
\hline Iris pseudacorus & E1 & . & . & 50 & Galeopsis bifida & E1 & . & 33 & . \\
\hline Galium palustre & E1 & . & . & 30 & Galium aparine & E1 & . & 33 & 30 \\
\hline Carex acuta & E1 & . & 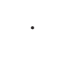 & 20 & Cirsium arvense & E1 & . & . & 40 \\
\hline Glyceria maxima & E1 & . & . & 20 & Lemnetea minoris & & & & \\
\hline Poa palustris & E1 & . & . & 10 & Lemna minor & E1 & . & 33 & . \\
\hline Filipendulo-Convolvuletea & & & & & Potametea pectinati & & & & \\
\hline Helianthus tuberosus & E1 & 17 & & . & Elodea canadensis & E1 & . & 33 & . \\
\hline Saponaria officinalis & E1 & 17 & 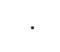 & . & Agropyretea & & & & \\
\hline Calystegia sepium & E1 & 8 & & 50 & intermedii-repentis & & & & \\
\hline Lythrum salicaria & E1 & 8 & 33 & 40 & Poa compressa & E1 & 17 & . & . \\
\hline Epilobium hirsutum & E1 & . & 66 & . & Elytrigia repens & E1 & . & . & 10 \\
\hline Mentha longifolia & E1 & . & 33 & . & Potentillion anserineae & & & & \\
\hline Myosoton aquaticum & E1 & . & 33 & 10 & Rorippa sylvestris & E1 & 42 & . & 10 \\
\hline Galega officinalis & E1 & . & 33 & . & Barbarea vulgaris & E1 & 25 & 66 & . \\
\hline Stachys palustris & E1 & . & . & 20 & Alopecuros geniculatus & E1 & . & 66 & . \\
\hline Echinocystis lobata & E1 & . & & 10 & Potentillo-Polygonetalia & & & & \\
\hline Bidentetea tripartitae & & & & & Agrostis stolonifera & E1 & 42 & 66 & . \\
\hline Rorippa palustris & E1 & 8 & & . & Plantago major & E1 & 25 & . & . \\
\hline Polygonum hydropiper & E1 & . & 100 & 30 & Rumex crispus & E1 & 8 & . & 10 \\
\hline Polygonum lapathifolium & E1 & & 33 & 20 & Ranunculus repens & E1 & 8 & . & . \\
\hline Ranunculus sceleratus & E1 & . & 33 & . & & & & & \\
\hline
\end{tabular}


Tab. 3. - continued

\begin{tabular}{|c|c|c|c|c|c|c|c|c|c|}
\hline & & & & & & & & & \\
\hline Succesive number & & 1 & 2 & 3 & Succesive number & & 1 & 2 & 3 \\
\hline Number of relevé & & 12 & 3 & 10 & Number of relevé & & 12 & 3 & 10 \\
\hline Author & & DSV & VP & $\mathrm{HU}$ & Author & & DSV & VP & $\mathrm{HU}$ \\
\hline$\underline{\text { Sign }}$ & & TL & $\mathrm{RP}$ & RP1 & $\underline{\text { Sign }}$ & & $\mathrm{TL}$ & $\mathrm{RP}$ & RP1 \\
\hline Molinietalia caeruleae & & & & & Alnion incanae & & & & \\
\hline Myosotis scorpioides & E1 & . & 100 & . & Rubus caesius & E2 & 75 & . & 10 \\
\hline Equisetum palustre & E1 & . & . & 10 & Ulmus laevis & $\mathrm{E} 2$ & 67 & . & . \\
\hline Scirpus sylvaticus & E1 & . & . & 10 & Alnus glutinosa & E2 & 17 & . & . \\
\hline Molinio-Arrhenatheretea & & & & & Solanum dulcamara & E1 & . & . & 20 \\
\hline Deschampsia cespitosa & E1 & 75 & . & . & Equisetum arvense & $\mathrm{E} 1$ & . & . & 10 \\
\hline Taraxacum officinale & E1 & 33 & . & . & Humulus lupulus & E1 & . & . & 10 \\
\hline Trifolium pratense & E1 & 33 & . & . & Fagetalia sylvaticae & & & & \\
\hline Plantago lanceolata & E1 & 33 & . & . & Carpinus betulus & E2 & 17 & . & . \\
\hline Achillea millefolium & E1 & 17 & . & . & Fraxinus excelsior & E2 & 8 & . & . \\
\hline Dactylis glomerata s.str. & E1 & 17 & . & . & Scrophularia nodosa & E1 & 8 & . & . \\
\hline Lotus corniculatus & E1 & 17 & . & . & Quercetalia pubescentis & & & & \\
\hline Galium mollugo & E1 & 8 & . & . & Campanula rapunculoides & $\mathrm{E} 1$ & 8 & . & . \\
\hline Ranunculus acris & E1 & 8 & . & 10 & Fraxinus ornus & $\mathrm{E} 1$ & 8 & . & . \\
\hline Veronica chamaedrys & E1 & 8 & . & . & Querco-Fagetea & & & & \\
\hline Leucanthemum ircutianum & E1 & 8 & . & . & Clematis vitalba & $\mathrm{E} 1$ & 8 & . & . \\
\hline Cerastium holosteoides & E1 & 8 & . & . & Carex digitata & E1 & 8 & . & . \\
\hline Poa trivialis & E1 & . & 66 & 50 & Rhamno-Prunetea & & & & \\
\hline Alopecurus pratensis & E1 & . & . & 20 & Crataegus monogyna & E2 & 33 & . & . \\
\hline Symphytum officinale & E1 & . & . & 20 & Rhamnus catharticus & E2 & 8 & . & . \\
\hline Lysimachia nummularia & $\mathrm{E} 1$ & . & . & 20 & Erico-Pinetea & & & & \\
\hline Carex hirta & E1 & . & . & 20 & Sesleria caerulea subsp. & E1 & 33 & . & . \\
\hline Potentilla anserina & E1 & . & . & 10 & calcaria & & & & \\
\hline Vicia cracca & E1 & & . & 10 & Chamaecytisus purpureus & E1 & 25 & . & . \\
\hline Festuco-Brometea s. lat. & & & & & Calamagrostis varia & E1 & 8 & . & . \\
\hline Peucedanum oreoselinum & E1 & 25 & . & . & Other species & & & & \\
\hline Genista tinctoria & E1 & 8 & . & . & Fallopia japonica & E1 & 8 & . & . \\
\hline Petrorhagia saxifraga & E1 & 8 & . & . & Poasp. & E1 & 8 & . & . \\
\hline Inula ensifolia & E1 & 8 & . & . & Agrostis sp. & $\mathrm{E} 1$ & 8 & . & . \\
\hline Salicetea purpureae & & & & & Physocarpus opulifolius & E2 & 8 & . & . \\
\hline Populus nigra & E2 & 83 & . & . & Galium rivale & E1 & . & . & 10 \\
\hline Salix alba & E2 & 33 & . & . & Mosses & & & & \\
\hline Salix eleagnos & E2 & 25 & . & . & Brachythecium rutabulum & E0 & 100 & . & . \\
\hline Salix purpurea & E2 & 58 & . & . & Cinclidotus fontinaloides & E0 & 58 & . & . \\
\hline Salix triandra & E2 & 8 & . & . & Tortella sp. & E0 & 67 & . & . \\
\hline Salix fragilis & E2 & 8 & . & & & & & & \\
\hline
\end{tabular}

1: Triseto-Leontodontetum - this paper; 2: Rorippo-Phalaridetum - Petrinac 1999; 3: Rorippo-Phalaridetum Hrivinák and Ujházy 2003 
DAKSKOBler I., SelišKar A., VReŠ B.
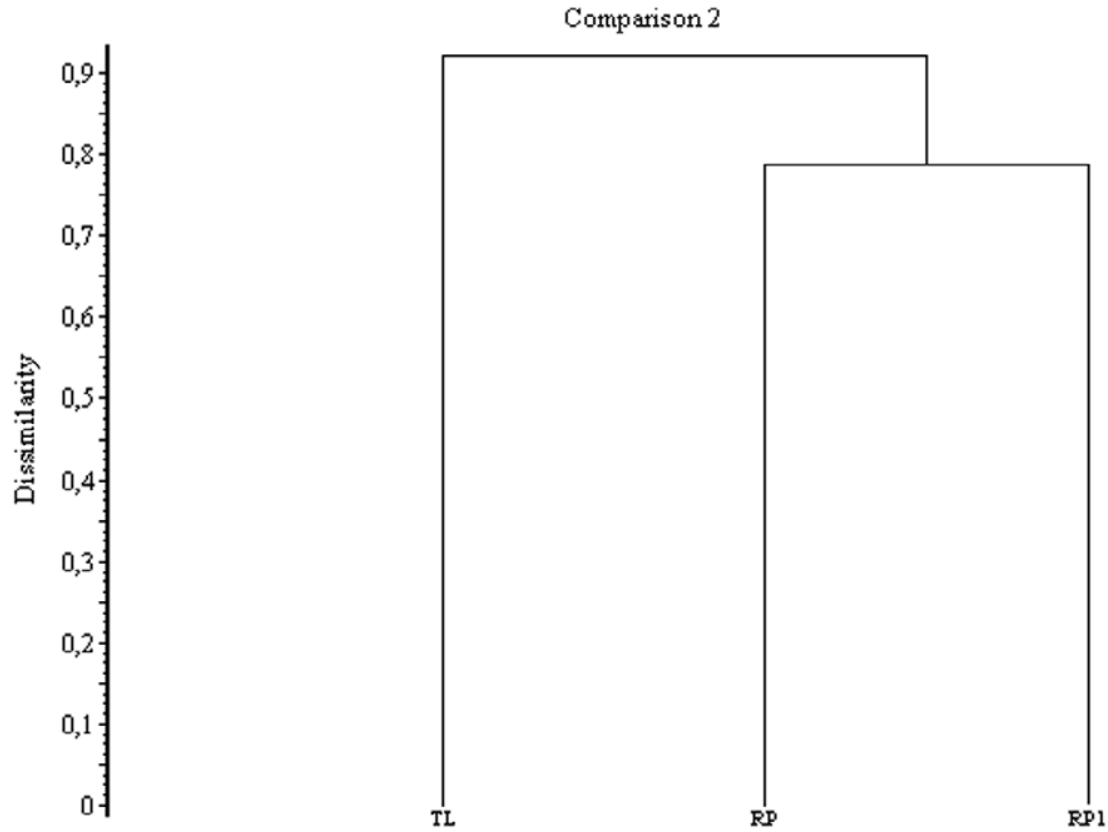

Fig. 8. Dendrogram of the two riparian communities (TL - Triseto-Leontodontetum, RP and RP1 Rorippo-Phalaridetum) - UPGMA, similarity ratio.

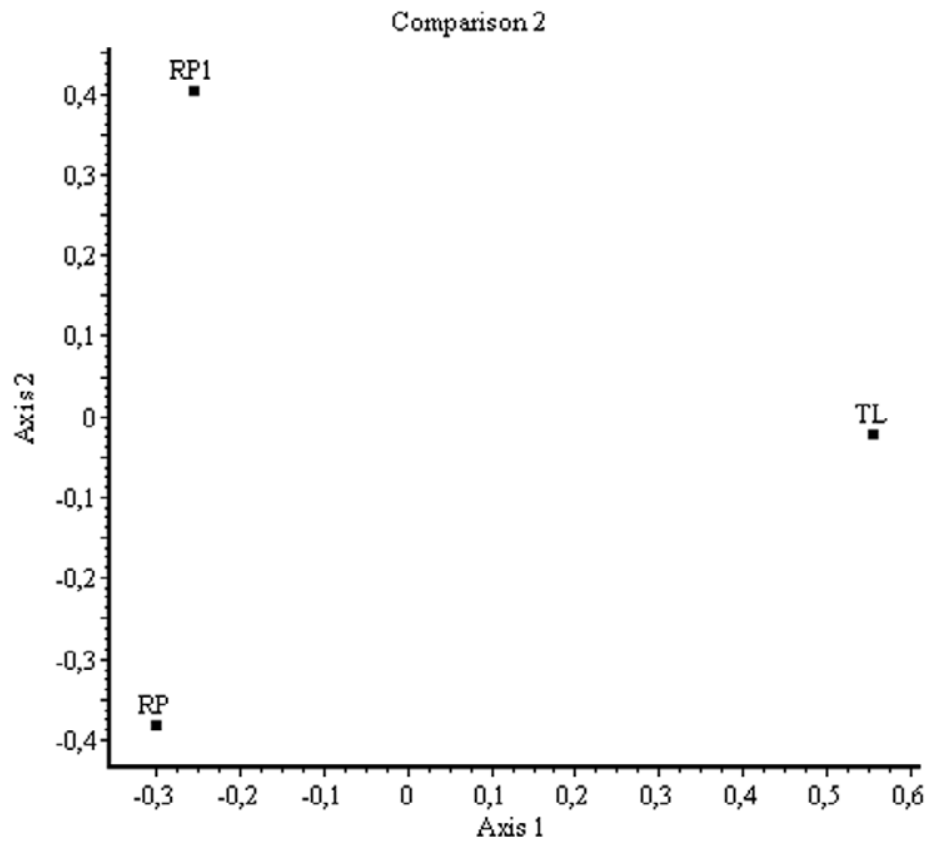

Fig. 9. Two-dimensional scatter-diagram of two riparian communities (TL - Triseto-Leontodontetum, RP and RP1 - Rorippo-Phalaridetum) - PCoA, similarity ratio. 
Diagnostic species of the new association are Leontodon brumatii (dominant species), Trisetum argenteum and some riparian mosses of which we established two Brachythecium rutabulum and Cinclidotus fontinaloides. Trisetum argenteum is a character species of scree communities, especially in the subalpine and alpine belts (AESCHIMANN et al. 2004). It is relatively frequently deposited and preserved on riparian rocks along the central or lower course of Alpine rivers, e.g. in Slovenia along the Soča and the Sava, so it is also a good indicator of the site or community of L. brumatii on riparian rocks. The nomenclature type of the new association, holotypus, is relevé No. 12 in table 1. The new association is subdivided into three variants: the typical (relevés 2 and 3 in table 1); the variant with Chamaecytisus purpureus (the differential species are also Peucedanum oreoselinum, Sesleria caerulea subsp. calcaria and Inula ensifolia) - relevés 4 to 6 in table 1- these stands indicate a possible transition into the community Leontodonti brumatii-Seslerietum calcariae described hereinafter, i.e. into the initial riparian grassland; and the variant with Deschampsia cespitosa (the differential species is also Phalaris arundinacea) - relevés 7 to 13 in table 1. Everywhere sand accumulates in the runnels carved into the rocks or in coarse gravel, conditions are created that facilitate successional development towards riparian willow stands with black poplar, which respond particularly well to sufficient amount of fine particles (BožIČ et al. 2008). The stands of this variant therefore indicate potential successional development into the community Salicetum eleagno-purpureae var. Populus nigra, into which relevés No. 14 and 15 were classified (see DAKSKOBLER 2010). Individual shoots of tree species Populus nigra, Ulmus laevis and Salix alba occur also in other relevés.

Most of the relevés in table 2 (the Soča valley) are classified into the new association Leontodonti brumatii-Seslerietum calcariae ass. nov. Its stands characterize the initial stony riparian grassland on undeveloped soil (lithosol). The species composition belongs to very different phytosociological groups (Tab. 4); the largest, some $21 \%$, is the

Tab. 4. Structure of the association Leontodonti brumatii-Selserietum calcariae according phytosociological groups (in \%).

\begin{tabular}{lc}
\hline Asplenietea trichomanis & 13 \\
Elyno-Seslerietea & 10 \\
Festuco-Brometea & 21 \\
Molinion caeruleae & 1,4 \\
Molinio-Arrhenatheretea & 1,4 \\
Scheuchzerio-Caricetea fuscae & 0,7 \\
Trifolio-Geranietea & 2,5 \\
Thlaspietea rotundifolii & 5,1 \\
Erico-Pinetea & 21 \\
Vaccinio-Piceetea & 0,7 \\
Mulgedio-Aconitetea & 2,2 \\
Quercetalia pubescentis & 9,5 \\
Fagetalia sylvaticae & 2,2 \\
Querco-Fagetea & 4,4 \\
Salicetea purpureae & 2,9 \\
Artemisietea vulgaris & 0,7 \\
Other species & 1,1 \\
\hline Total & 100 \\
\hline
\end{tabular}


proportion of species of dry grasslands from the class Festuco-Brometea and species of basophilic pine forests from the class Erico-Pinetea, followed by chasmophytic species (Asplenietea trichomanis), species of subalpine grasslands (Elyno-Seslerietea) and thermophilic oak forests (Quercetalia pubescentis), which have a proportion of $10 \%$. Varied species composition also indicates a certain similarity with the communities of thermophilic forest edges from the class Trifolio-Geranietea, although it features a small proportion of diagnostic species of this class $(2.5 \%)$. In support of the synsystematic classification a synoptic table was made (Tab. 5), into which two riparian meadow communities - Centaureo dichroanthae-Globularietum cordifoliae Pignatti 1953 (FEOLI

Tab. 5. Synoptic table of pioneer grassland and fringe vegetation.

\begin{tabular}{|c|c|c|c|c|c|c|}
\hline Succesive number & & 1 & 2 & 3 & 4 & 5 \\
\hline Number of relevé & & 12 & 14 & 9 & 21 & 10 \\
\hline Author & & DSV & CFP & $\mathrm{DZ}$ & GKL & $\mathrm{AC}$ \\
\hline Sign & & LS & CG & GB & $\mathrm{CP}$ & GP \\
\hline \multicolumn{7}{|l|}{ Asplenietea trichomanis } \\
\hline Leontodon hispidus subsp. brumatii & E1 & 100 & . & . & . & . \\
\hline Hieracium porrifolium & E1 & 75 & 36 & . & . & . \\
\hline Athamanta turbith & E1 & 50 & . & . & . & . \\
\hline Saxifraga crustata & E1 & 33 & . & . & . & . \\
\hline Phyteuma scheuchzeri subsp. columnae & E1 & 25 & . & . & . & . \\
\hline Asplenium ruta-muraria & E1 & 8 & . & . & . & . \\
\hline Paederota lutea & E1 & 8 & . & . & . & . \\
\hline Seseli gouanii & E1 & . & 43 & . & . & . \\
\hline Dianthus sylvestris & E1 & . & 7 & . & . & 10 \\
\hline Silene hayekiana & E1 & . & . & 33 & . & . \\
\hline Elyno-Seslerietea & & . & . & . & . & . \\
\hline Sesleria caerulea subsp. calcaria & E1 & 100 & 86 & & & \\
\hline Aster bellidiastrum & E1 & 67 & 7 & . & . & . \\
\hline Globularia cordifolia & E1 & 25 & 100 & 33 & . & . \\
\hline Rhinanthus aristatus & E1 & 25 & 14 & 11 & . & . \\
\hline Erigeron glabratus & E1 & 8 & . & . & . & . \\
\hline Phyteuma orbiculare & E1 & 8 & . & . & . & . \\
\hline Carex mucronata & E1 & . & 93 & . & . & . \\
\hline Helianthemum alpestre & E1 & . & 36 & . & . & . \\
\hline Dryas octopetala & E1 & . & 14 & . & . & . \\
\hline Gentiana clusii & E1 & . & 7 & . & . & . \\
\hline Carlina acaulis s. lat. & E1 & . & 7 & 89 & . & 20 \\
\hline Helianthemum nummularium subsp. grandiflorum & E1 & . & . & 89 & . & . \\
\hline Ranunculus carinthiacus & E1 & . & . & 56 & . & . \\
\hline Betonica alopecuros & E1 & . & . & 44 & . & . \\
\hline Galium anisophyllon & E1 & . & . & 33 & . & . \\
\hline Alchemilla glaucescens & E1 & . & . & 22 & . & . \\
\hline Gentiana verna & E1 & . & . & 11 & . & . \\
\hline
\end{tabular}


Tab. 5. - continued

\begin{tabular}{|c|c|c|c|c|c|c|}
\hline Succesive number & & 1 & 2 & 3 & 4 & 5 \\
\hline Number of relevé & & 12 & 14 & 9 & 21 & 10 \\
\hline Author & & DSV & $\mathrm{CFP}$ & $\mathrm{DZ}$ & GKL & $\mathrm{AC}$ \\
\hline Sign & & $\mathrm{LS}$ & $\mathrm{CG}$ & GB & $\mathrm{CP}$ & GP \\
\hline Hieracium valdepilosum & E1 & . & . & 11 & . & . \\
\hline Hieracium villosum & E1 & . & . & 11 & . & . \\
\hline Festuca calva & E1 & . & . & 11 & . & . \\
\hline Phyteuma orbiculare & E1 & . & . & 11 & . & . \\
\hline \multicolumn{7}{|l|}{ Festuco-Brometea } \\
\hline Carex humilis & E1 & 58 & 86 & 11 & 70 & 10 \\
\hline Centaurea scabiosa (inc. subsp. fritschii) & E1 & 58 & . & 44 & 10 & 70 \\
\hline Genista tinctoria & E1 & 58 & . & 11 & 10 & 10 \\
\hline Peucedanum oreoselinum & E1 & 42 & 93 & 56 & 70 & 60 \\
\hline Inula ensifolia & E1 & 33 & 86 & . & . & 10 \\
\hline Euphorbia cyparissias & E1 & 33 & 21 & 44 & 50 & 90 \\
\hline Satureja montana subsp. variegata & E1 & 25 & 14 & . & 10 & . \\
\hline Stachys recta & E1 & 25 & . & . & 30 & 50 \\
\hline Buphthalmum salicifolium & E1 & 25 & 7 & . & 50 & 80 \\
\hline Bromopsis erecta & E1 & 17 & . & 100 & 50 & 30 \\
\hline Inula hirta & E1 & 17 & . & . & 50 & 10 \\
\hline Ononis spinosa & E1 & 17 & . & 22 & . & . \\
\hline Medicago falcata & E1 & 17 & . & . & 40 & 10 \\
\hline Gymnadenia conopsea & E1 & 17 & 29 & 78 & 10 & . \\
\hline Koeleria pyramidata & E1 & 8 & . & 100 & 30 & 40 \\
\hline Thymus praecox s. lat. & E1 & 8 & 7 & 78 & 10 & 50 \\
\hline Salvia pratensis & E1 & 8 & . & 78 & 30 & 70 \\
\hline Trifolium montanum & E1 & 8 & . & 56 & 50 & 20 \\
\hline Allium carinatum s. lat. & E1 & 7 & . & 44 & . & 30 \\
\hline Centaurea dichroantha & E1 & . & 100 & . & . & . \\
\hline Teucrium montanum & E1 & . & 93 & 11 & 10 & 10 \\
\hline Fumana procumbens & E1 & . & 93 & . & . & . \\
\hline Stipa eriocaulis & E1 & . & 93 & . & 10 & . \\
\hline Galium lucidum & E1 & . & 93 & . & 30 & . \\
\hline Plantago holosteum & E1 & . & 86 & 67 & . & . \\
\hline Thesium divaricatum & E1 & . & 71 & . & . & . \\
\hline Bromopsis condensata & E1 & . & 71 & . & . & . \\
\hline Genista sericea & E1 & . & 64 & . & . & . \\
\hline Thymus longicaulis & E1 & . & 64 & . & . & . \\
\hline Chrysopogon gryllus & E1 & . & 64 & . & 10 & 10 \\
\hline Helianthemuт nuтmularium subsp. obsurum & E1 & . & 64 & . & 10 & 40 \\
\hline Scorzonera austriaca & E1 & . & 57 & . & 50 & . \\
\hline Linum tenuifolium & E1 & . & 50 & . & . & . \\
\hline Cytisus pseudoprocumbens & E1 & . & 50 & . & . & . \\
\hline Potentilla australis & E1 & . & 43 & . & . & . \\
\hline Leontodon crispus & E1 & . & 43 & . & 10 & . \\
\hline
\end{tabular}


Dakskobler I., SelišKar A., VReŠ B.

Tab. 5. - continued

\begin{tabular}{|c|c|c|c|c|c|c|}
\hline Succesive number & & 1 & 2 & 3 & 4 & 5 \\
\hline Number of relevé & & 12 & 14 & 9 & 21 & 10 \\
\hline Author & & DSV & CFP & $\mathrm{DZ}$ & GKL & $\mathrm{AC}$ \\
\hline$\underline{\text { Sign }}$ & & LS & CG & GB & $\mathrm{CP}$ & GP \\
\hline Asperula cynanchica & E1 & . & 43 & 22 & 10 & 70 \\
\hline Orchis morio & E1 & . & 43 & . & . & . \\
\hline Scabiosa graminifolia & E1 & . & 36 & . & . & . \\
\hline Dianthus monspessulanus & E1 & . & 36 & . & 10 & 10 \\
\hline Hippocrepis comosa & E1 & . & 36 & 78 & 10 & . \\
\hline Teucrium chamaedrys & E1 & . & 29 & . & 30 & 90 \\
\hline Plantago argeneta & E1 & . & 21 & 33 & 30 & . \\
\hline Sanguisorba muricata (inc. S. minor) & E1 & . & 21 & 100 & 10 & 40 \\
\hline Galium verum & E1 & . & 21 & 89 & . & 80 \\
\hline Carex liparocarpos & E1 & . & 14 & . & . & . \\
\hline Asperula purpurea & E1 & . & 14 & . & . & . \\
\hline Scabiosa triandra & E1 & . & 14 & 67 & . & 40 \\
\hline Festuca rupiciola (inc. F. pseudovina, F. ovina agg.) & E1 & . & 14 & 100 & 30 & 50 \\
\hline Anacamptys pyramidalis & E1 & . & 14 & . & . & . \\
\hline Carex caryophyllea & E1 & . & 14 & 78 & . & . \\
\hline Hypochoeris maculata & E1 & . & 14 & . & . & 10 \\
\hline Globularia elongata & E1 & . & 14 & . & . & . \\
\hline Allium senescens & E1 & . & 14 & 11 & . & . \\
\hline Botriochloa ishaemum & E1 & . & 7 & . & . & . \\
\hline Ophrys apifera & E1 & . & 7 & . & . & . \\
\hline Centaurea bracteata (C. gaudinii) & E1 & . & 7 & . & . & . \\
\hline Betonica serotina & E1 & . & 7 & . & . & . \\
\hline Gentianella pilosa & E1 & . & 7 & 56 & . & . \\
\hline Hieracium pilosella & E1 & . & 7 & 11 & 10 & . \\
\hline Brachypodium rupestre & E1 & . & . & 100 & 70 & 80 \\
\hline Pimpinella saxifraga & E1 & . & . & 100 & 30 & 60 \\
\hline Plantago media & E1 & . & . & 100 & 10 & 30 \\
\hline Prunella grandiflora & E1 & . & . & 89 & 10 & 20 \\
\hline Briza media & E1 & . & . & 89 & 30 & 30 \\
\hline Medicago lupulina & E1 & . & . & 89 & 10 & 20 \\
\hline Polygala comosa & E1 & . & . & 89 & . & . \\
\hline Silene vulgaris subsp. vulgaris & E1 & . & . & 89 & . & 20 \\
\hline Orchis ustulata & E1 & . & . & 89 & . & . \\
\hline Campanula rotundifolia & E1 & . & . & 79 & . & . \\
\hline Campanula glomerata & E1 & . & . & 78 & . & . \\
\hline Orchis militaris & E1 & . & . & 67 & . & . \\
\hline Linum catharticum & E1 & . & . & 56 & . & 20 \\
\hline Ranunculus bulbosus & E1 & . & . & 44 & . & . \\
\hline Euphrasia stricta & E1 & . & . & 22 & . & . \\
\hline Potentilla pusilla & E1 & . & . & 11 & . & . \\
\hline Arabis hirsuta & E1 & & . & 11 & 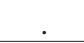 & . \\
\hline
\end{tabular}


Tab. 5. - continued

\begin{tabular}{|c|c|c|c|c|c|c|}
\hline Succesive number & & 1 & 2 & 3 & 4 & 5 \\
\hline Number of relevé & & 12 & 14 & 9 & 21 & 10 \\
\hline Author & & DSV & CFP & $\mathrm{DZ}$ & GKL & $\mathrm{AC}$ \\
\hline Sign & & LS & $\mathrm{CG}$ & GB & $\mathrm{CP}$ & GP \\
\hline Astragalus onobrychis & E1 & . & . & 11 & . & . \\
\hline Cuscuta epithimum & E1 & . & . & 11 & . & 10 \\
\hline Carlina vulgaris & E1 & . & . & 11 & . & . \\
\hline Filipendula vulgaris & E1 & . & . & 11 & 70 & 60 \\
\hline Orobanche gracilis & E1 & . & . & 11 & . & . \\
\hline Gentianella ciliata & E1 & . & . & 11 & . & . \\
\hline Rhinanthus freynii & E1 & . & . & 11 & . & . \\
\hline Hypochoeris maculata & E1 & . & . & 11 & 30 & 10 \\
\hline Cirsium pannonicum & E1 & . & . & . & 70 & 30 \\
\hline Euphorbia verrucosa & E1 & . & . & . & 70 & 30 \\
\hline Knautia illyrica & E1 & . & . & . & 50 & . \\
\hline Dorycnium germanicum & E1 & . & . & . & 50 & 60 \\
\hline Vicia tenuifolia & E1 & . & . & . & 30 & . \\
\hline Genista sylvestris & E1 & . & . & . & 30 & . \\
\hline Centaurea rupestris & E1 & . & . & . & 30 & . \\
\hline Scorzonera villosa & E1 & . & . & . & 30 & . \\
\hline Veronica barrelieri & E1 & . & . & . & 10 & . \\
\hline Euphorbia nicaeensis & E1 & . & . & . & 10 & . \\
\hline Thesium linophyllum & E1 & . & . & . & 10 & . \\
\hline Pulsatilla montana & E1 & . & . & . & 10 & . \\
\hline Lychnis viscaria & E1 & . & . & . & . & 60 \\
\hline Scabiosa hladnikiana & E1 & . & . & . & . & 30 \\
\hline Prunella laciniata & E1 & . & . & . & . & 20 \\
\hline Cuscuta epithymum & E1 & . & . & . & . & 20 \\
\hline Ornithogalum sphaerocarpum & E1 & . & . & . & . & 20 \\
\hline Anthyllis vulneraria & E1 & . & . & . & . & 10 \\
\hline \multicolumn{7}{|l|}{ Thero-Brachypodietea } \\
\hline Koeleria lobata & E1 & . & 100 & . & . & . \\
\hline Artemisia alba & E1 & . & 29 & . & . & . \\
\hline Eryngium amethystinum & E1 & . & 21 & . & . & 10 \\
\hline Campanula sibirica & E1 & . & 21 & . & . & . \\
\hline Medicago prostrata & E1 & . & 7 & . & . & . \\
\hline Melica ciliata & E1 & . & 7 & . & . & . \\
\hline \multicolumn{7}{|l|}{ Koelerio-Corynephoretea } \\
\hline Cardaminopsis arenosa & E1 & . & . & 33 & . & . \\
\hline Sedum sexangulare & E1 & . & . & 22 & . & . \\
\hline Cerastium brachypetalum & E1 & . & . & 22 & . & . \\
\hline Petrorhagia saxifraga & E1 & . & . & 11 & . & . \\
\hline \multicolumn{7}{|l|}{ Molinion caeruleae } \\
\hline Laserpitium prutenicum & E1 & 33 & 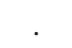 & . & . & . \\
\hline Molinia caerulea subsp. caerulea & E1 & . & & 11 & . & . \\
\hline
\end{tabular}


Dakskobler I., SelišKar A., VReŠ B.

Tab. 5. - continued

\begin{tabular}{|c|c|c|c|c|c|c|}
\hline Succesive number & & 1 & 2 & 3 & 4 & 5 \\
\hline Number of relevé & & 12 & 14 & 9 & 21 & 10 \\
\hline Author & & DSV & CFP & $\mathrm{DZ}$ & GKL & $\mathrm{AC}$ \\
\hline$\underline{\text { Sign }}$ & & LS & $\mathrm{CG}$ & GB & $\mathrm{CP}$ & GP \\
\hline Carex distans & E1 & . & . & 11 & . & . \\
\hline Carex tomentosa & E1 & . & . & 11 & . & . \\
\hline Sanguisorba officinalis & E1 & . & . & 11 & . & . \\
\hline Herminium monorchis & E1 & . & . & 11 & . & . \\
\hline Inula salicina & E1 & . & . & . & 10 & 20 \\
\hline Selinum carvifolia & E1 & . & . & . & . & 10 \\
\hline \multicolumn{7}{|l|}{ Molinio-Arrhenatheretea } \\
\hline Taraxacum officinale & E1 & 25 & . & . & . & . \\
\hline Lotus corniculatus agg. & E1 & 8 & 29 & 89 & 50 & 30 \\
\hline Dactylis glomerata & E1 & . & 29 & 89 & 10 & 80 \\
\hline Senecio jacobea & E1 & . & 14 & . & 10 & . \\
\hline Leontodon hispidus subsp. hispidus & E1 & . & . & 100 & . & 10 \\
\hline Trifolium pratense & E1 & . & . & 89 & . & . \\
\hline Centaurea jacea & E1 & . & . & 78 & 10 & 70 \\
\hline Achillea millefolium & E1 & . & . & 78 & . & 50 \\
\hline Galium mollugo (inc. G. album) & E1 & . & . & 78 & . & 30 \\
\hline Helictotrichon pubescens & E1 & . & . & 78 & . & . \\
\hline Prunella vulgaris & E1 & . & . & 78 & . & . \\
\hline Rhinanthus minor & E1 & . & . & 67 & . & 10 \\
\hline Trifolium repens & E1 & . & . & 67 & . & . \\
\hline Vicia cracca & E1 & . & . & 67 & . & . \\
\hline Leucanthemum ircutianum (inc. L. vulgare) & E1 & . & . & 67 & 10 & 30 \\
\hline Plantago lanceolata & E1 & . & . & 56 & . & . \\
\hline Orchis coriophora subsp. coriophora & E1 & . & . & 56 & . & . \\
\hline Ranunculus acris & E1 & . & . & 44 & . & . \\
\hline Euphrasia rostkoviana & E1 & . & . & 44 & . & . \\
\hline Lathyrus pratensis & E1 & . & . & 44 & 10 & 40 \\
\hline Leontodon autumnalis & E1 & . & . & 44 & . & . \\
\hline Tragopogon pratensis & E1 & . & . & 44 & . & 10 \\
\hline Ranunculus nemorosus & E1 & . & . & 33 & . & . \\
\hline Rumex acetosa & E1 & . & . & 33 & . & . \\
\hline Luzula campestris & E1 & . & . & 33 & . & . \\
\hline Festuca pratensis & E1 & . & . & 22 & . & . \\
\hline Achillea roseoalba & E1 & 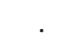 & . & 22 & . & . \\
\hline Carex hirta & E1 & . & . & 11 & . & . \\
\hline Trifolium campestre & E1 & . & . & 11 & . & . \\
\hline Knautia arvensis & E1 & . & . & 11 & . & 30 \\
\hline Festuca arundinacea & E1 & . & . & 11 & . & . \\
\hline Heracleum sphondylium & E1 & . & . & 11 & . & . \\
\hline Pimpinella major & E1 & . & . & 11 & . & . \\
\hline Allium scorodoprasum & E1 & & . & 11 & . & 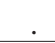 \\
\hline
\end{tabular}


Tab. 5. - continued

\begin{tabular}{|c|c|c|c|c|c|c|}
\hline Succesive number & & 1 & 2 & 3 & 4 & 5 \\
\hline Number of relevé & & 12 & 14 & 9 & 21 & 10 \\
\hline Author & & DSV & CFP & $\mathrm{DZ}$ & GKL & $\mathrm{AC}$ \\
\hline$\underline{\text { Sign }}$ & & LS & CG & GB & $\mathrm{CP}$ & GP \\
\hline Stellaria graminea & E1 & . & . & 11 & . & . \\
\hline Bromus hordeaceus & E1 & . & . & 11 & . & . \\
\hline Veronica chamaedrys & E1 & . & . & . & 10 & 20 \\
\hline Arrhenatherum elatius & E1 & . & . & . & 10 & . \\
\hline Knautia arvensis & E1 & . & . & . & . & 30 \\
\hline Festuca rubra & E1 & . & . & . & . & 30 \\
\hline Poa angustifolia & E1 & . & . & . & . & 20 \\
\hline Rumex acetosa & E1 & . & . & . & . & 10 \\
\hline Trisetum flavescens & E1 & . & . & . & . & 10 \\
\hline \multicolumn{7}{|l|}{ Calluno-Ulicetea } \\
\hline Potentilla erecta & E1 & . & . & 78 & . & 10 \\
\hline Chamaecytisus supinus & E1 & . & . & . & 30 & . \\
\hline Chamaespartium sagittale & E1 & . & . & . & 10 & . \\
\hline Genista germanica & E1 & . & . & . & . & 10 \\
\hline Polygala vulgaris & E1 & . & . & . & . & 10 \\
\hline \multicolumn{7}{|c|}{ Scheuchzerio-Caricetea fuscae } \\
\hline Parnassia palustris & E1 & 17 & . & 33 & . & . \\
\hline Schoenus nigricans & E1 & . & 79 & . & . & . \\
\hline Carex panicea & E1 & . & . & 11 & . & . \\
\hline \multicolumn{7}{|l|}{ Trifolio-Geranietea } \\
\hline Peucedanum cervaria & E1 & 42 & . & . & 100 & 100 \\
\hline Vincetoxicum hirundinaria & E1 & 8 & 7 & 22 & 30 & 20 \\
\hline Lilium bulbiferum & E1 & 8 & . & . & . & . \\
\hline Anthericum ramosum & E1 & . & 43 & . & 50 & 80 \\
\hline Silene nutans & E1 & . & . & 89 & 30 & 30 \\
\hline Thalictrum minus & E1 & . & . & 33 & 70 & 40 \\
\hline Valeriana collina & E1 & . & . & 11 & . & 10 \\
\hline Ferulago galbanifera & E1 & . & . & . & 90 & . \\
\hline Dictamnus albus & E1 & . & . & . & 70 & . \\
\hline Iris illyrica & E1 & . & . & . & 50 & . \\
\hline Geranium sanguineum & E1 & . & . & . & 50 & 70 \\
\hline Polygonatum odoratum & E1 & . & . & . & 50 & 10 \\
\hline Paeonia officinalis & E1 & . & . & . & 30 & . \\
\hline Trifolium alpestre & E1 & . & . & . & 30 & . \\
\hline Viola hirta & E1 & . & . & . & 30 & 70 \\
\hline Trifolium rubens & E1 & . & . & . & 30 & 50 \\
\hline Coronilla coronata & E1 & . & . & . & 20 & . \\
\hline Trifolium medium & E1 & . & . & . & 10 & 20 \\
\hline Ruta divaricata & E1 & . & . & . & 10 & . \\
\hline Verbascum austriacum & E1 & . & . & . & 10 & . \\
\hline Lathyrus latifolius & E1 & & . & . & 10 & . \\
\hline
\end{tabular}


Dakskobler I., SelišKar A., VReŠ B.

Tab. 5. - continued

\begin{tabular}{|c|c|c|c|c|c|c|}
\hline Succesive number & & 1 & 2 & 3 & 4 & 5 \\
\hline Number of relevé & & 12 & 14 & 9 & 21 & 10 \\
\hline Author & & DSV & CFP & $\mathrm{DZ}$ & GKL & $\mathrm{AC}$ \\
\hline Sign & & LS & CG & GB & $\mathrm{CP}$ & GP \\
\hline Lilium bulbiferum & E1 & . & . & . & 10 & . \\
\hline Hypericum perforatum & E1 & . & . & . & 10 & . \\
\hline Potentilla recta & E1 & . & . & . & 10 & . \\
\hline Origanum vulgare & E1 & . & . & . & . & 70 \\
\hline Clinopodium vulgare & E1 & . & . & . & . & 50 \\
\hline Coronilla varia & E1 & . & . & . & . & 20 \\
\hline Rosa gallica & E1 & . & . & . & . & 20 \\
\hline Veronica jacquiniii & E1 & . & . & . & . & 10 \\
\hline Melampyrum nemorosum & E1 & . & . & . & . & 10 \\
\hline Dianthus barbatus & E1 & . & . & . & . & 10 \\
\hline Inula conyza & E1 & . & . & . & . & 10 \\
\hline Veronica teucrium & E1 & . & . & . & . & 10 \\
\hline Lathyrus sylvestris & E1 & . & . & . & . & 10 \\
\hline Vicia tenuifolia & E1 & . & . & . & . & 10 \\
\hline \multicolumn{7}{|l|}{ Thlaspietea rotundifolii } \\
\hline Petasites paradoxus & E1 & 33 & 7 & 22 & . & . \\
\hline Campanula cespitosa & E1 & 25 & 14 & . & . & . \\
\hline Peucedanum verticillare & E1 & 17 & . & . & . & . \\
\hline Biscutella laevigata & E1 & 17 & 50 & 78 & . & . \\
\hline Achnatherum calamagrostis & E1 & 8 & 14 & . & . & . \\
\hline Trisetum argenteum & E1 & 8 & . & . & . & . \\
\hline Silene vulgaris subsp. glareosa & E1 & 8 & 7 & . & . & . \\
\hline Euphorbia triflora subsp. kerneri & E1 & . & 93 & . & . & . \\
\hline Gypsophila repens & E1 & . & 79 & . & . & . \\
\hline Trinia glauca & E1 & . & 71 & . & . & . \\
\hline Matthiola carnica & E1 & . & 57 & . & . & . \\
\hline Hieracium piloselloides & E1 & . & 50 & 22 & . & . \\
\hline Brassica glabrescens & E1 & . & 36 & . & . & . \\
\hline Crambe tataria & E1 & . & 36 & . & . & . \\
\hline Reseda lutea & E1 & . & 21 & . & . & . \\
\hline Diplotaxis tenuifolia & E1 & . & 21 & . & . & . \\
\hline Euphrasia cuspidata & E1 & . & 14 & . & . & . \\
\hline Rumex scutatus & E1 & . & 7 & . & . & . \\
\hline Rumex scutatus & E1 & . & . & 33 & . & . \\
\hline Hieracium glaucum & E1 & . & . & 11 & . & . \\
\hline Dianthus sternbergii & E1 & . & . & 11 & . & . \\
\hline \multicolumn{7}{|l|}{ Erico-Pinetea } \\
\hline Calamagrostis varia & E1 & 83 & . & 11 & 10 & 10 \\
\hline Chamaecytisus purpureus & E1 & 67 & 57 & 11 & . & . \\
\hline Allium ericetorum & E1 & 50 & 7 & . & . & . \\
\hline Erica carnea & E1 & 42 & 93 & . & . & 20 \\
\hline
\end{tabular}


Tab. 5. - continued

\begin{tabular}{|c|c|c|c|c|c|c|}
\hline Succesive number & & 1 & 2 & 3 & 4 & 5 \\
\hline Number of relevé & & 12 & 14 & 9 & 21 & 10 \\
\hline Author & & DSV & CFP & $\mathrm{DZ}$ & GKL & $\mathrm{AC}$ \\
\hline Sign & & $\mathrm{LS}$ & $\mathrm{CG}$ & GB & $\mathrm{CP}$ & GP \\
\hline Aster amellus & E1 & 50 & & 11 & . & 10 \\
\hline Leontodon incanus & $\mathrm{E} 1$ & 42 & 7 & . & . & . \\
\hline Carex ornithopoda & E1 & 33 & . & . & . & . \\
\hline Epipactis atrorubens & E1 & 25 & . & 11 & . & . \\
\hline Pinus nigra & E2 & 25 & . & . & . & . \\
\hline Polygala chamaebuxus & E1 & 25 & 7 & . & . & . \\
\hline Chamaecytisus hirsutus & E2 & 17 & . & . & . & 20 \\
\hline Molinia caerulea subsp. arundinacea & E1 & 8 & 7 & 11 & 30 & . \\
\hline Pinus sylvestris & E2 & 8 & . & . & . & . \\
\hline Polygala nicaeensis subsp. forojulensis & E1 & . & 43 & 11 & 10 & . \\
\hline Daphne cneorum & E1 & . & 14 & . & . & . \\
\hline Genista januensis & E1 & . & . & . & . & 10 \\
\hline \multicolumn{7}{|l|}{ Vaccinio-Piceetea } \\
\hline Solidago virgaurea & E1 & 8 & . & . & 10 & 60 \\
\hline Picea abies & $\mathrm{E} 2 \mathrm{a}$ & 8 & . & 11 & . & . \\
\hline \multicolumn{7}{|l|}{ Mulgedio-Aconitetea } \\
\hline Salix appendiculata & $\mathrm{E} 2 \mathrm{a}$ & 50 & . & . & . & . \\
\hline \multicolumn{7}{|l|}{ Rhamno-Prunetea } \\
\hline Cornus sanguinea & E1 & . & . & . & 30 & . \\
\hline Prunus mahaleb & E1 & . & . & . & 20 & . \\
\hline Crataegus monogyna & E1 & . & . & . & 10 & . \\
\hline Rhamnus rupestris & E1 & . & . & . & 10 & . \\
\hline Prunus spinosa & $\mathrm{E} 1$ & . & . & . & 10 & . \\
\hline Rosa canina & E1 & . & . & . & 10 & . \\
\hline Rubus fruticosus agg. & E1 & . & . & . & . & 10 \\
\hline \multicolumn{7}{|l|}{ Quercetalia pubescentis } \\
\hline Fraxinus ornus & $\mathrm{E} 2 \mathrm{a}$ & 75 & 7 & . & 30 & . \\
\hline Ostrya carpinifolia & $\mathrm{E} 2 \mathrm{a}$ & 33 & 7 & . & 10 & 30 \\
\hline Lembotropis nigricans & E1 & 25 & . & . & 10 & 20 \\
\hline Carex flacca & E1 & 25 & . & 22 & . & 70 \\
\hline Campanula rapunculoides & E1 & 17 & . & . & 10 & 20 \\
\hline Clematis recta & E1 & 17 & 7 & . & . & 10 \\
\hline Coronilla emerus subsp. emeroides & E1 & 8 & . & . & . & . \\
\hline Sorbus aria & $\mathrm{E} 2 \mathrm{a}$ & 8 & . & . & 10 & . \\
\hline Coronilla emerus subsp. emerus & $\mathrm{E} 2 \mathrm{a}$ & 8 & . & . & . & . \\
\hline Primula veris subsp. columnae & E1 & . & . & 89 & . & . \\
\hline Helleborus multifidus subsp. istriacus & E1 & . & . & . & 50 & . \\
\hline Sesleria autumnalis & E1 & . & . & . & 30 & . \\
\hline Mercurialis ovata & E1 & . & . & . & 30 & . \\
\hline Melittis melissophyllum & E1 & . & . & . & 30 & 40 \\
\hline Lathyrus niger & E1 & . & & . & 20 & 10 \\
\hline
\end{tabular}


Dakskobler I., SelišKar A., VReŠ B.

Tab. 5. - continued

\begin{tabular}{|c|c|c|c|c|c|c|}
\hline Succesive number & & 1 & 2 & 3 & 4 & 5 \\
\hline Number of relevé & & 12 & 14 & 9 & 21 & 10 \\
\hline Author & & DSV & CFP & DZ & GKL & $\mathrm{AC}$ \\
\hline$\underline{\text { Sign }}$ & & LS & $\mathrm{CG}$ & GB & $\mathrm{CP}$ & GP \\
\hline Cnidium silaifolium & E1 & . & . & . & 10 & . \\
\hline Quercus pubescens & E1 & . & . & . & 10 & . \\
\hline Hypericum montanum & E1 & . & . & . & 10 & 20 \\
\hline Tanacetum corymbosum & E1 & . & . & . & . & 20 \\
\hline Buglossoides purpurocaerulea & E1 & . & . & . & . & 20 \\
\hline Campanula persicifolia & E1 & . & . & . & . & 10 \\
\hline Quercus cerris & E1 & . & . & . & . & 10 \\
\hline Calamintha menthifolia & E1 & . & . & . & . & 10 \\
\hline \multicolumn{7}{|l|}{ Fagetalia sylvaticae } \\
\hline Tilia cordata & E2 & 17 & . & . & . & . \\
\hline Anemone trifolia & E1 & 8 & . & . & . & . \\
\hline Lathyrus vernus & E1 & 8 & . & . & . & . \\
\hline Ranunculus lanuginosus & E1 & 8 & . & . & . & . \\
\hline Fagus sylvatica & E2 & 8 & . & . & . & . \\
\hline Knautia drymeia & E1 & . & . & 11 & . & 30 \\
\hline Cyclamen purpurascens & E1 & . & . & . & 10 & . \\
\hline Salvia glutinosa & E1 & . & . & . & . & 20 \\
\hline \multicolumn{7}{|l|}{ Querco-Fagetea } \\
\hline Clematis vitalba & E2 & 42 & . & . & 10 & 10 \\
\hline Carex digitata & E1 & 17 & . & . & . & . \\
\hline Ulmus minor & E1 & 8 & . & . & . & . \\
\hline Pyrus pyraster & E2 & 8 & . & . & . & . \\
\hline Listera ovata & E1 & 8 & . & 11 & . & . \\
\hline Corylus avellana & E1 & 8 & . & . & 20 & . \\
\hline Quercus robur & E1 & 8 & . & . & . & . \\
\hline Cruciata glabra & E1 & . & . & 67 & . & 20 \\
\hline Potentilla alba & E1 & . & . & . & 70 & . \\
\hline Betonica officinalis & E1 & . & . & . & 70 & 70 \\
\hline Serratula tinctoria & E1 & . & . & . & 30 & 30 \\
\hline Hepatica nobilis & E1 & . & . & . & 10 & . \\
\hline Carex montana & E1 & . & . & . & 10 & . \\
\hline Helleborus odorus & E1 & . & . & . & . & 30 \\
\hline Festuca heterophylla & E1 & . & . & . & . & 20 \\
\hline Hieracium racemosum & E1 & . & . & . & . & 10 \\
\hline Pteridium aquilinum & E1 & . & . & . & . & 10 \\
\hline Hedera helix & E1 & . & . & . & . & 10 \\
\hline \multicolumn{7}{|l|}{ Salicetea purpureae } \\
\hline Salix eleagnos & E2 & 25 & 7 & . & . & . \\
\hline Salix purpurea & E2 & 25 & . & . & . & . \\
\hline Populus nigra & E2 & 17 & . & . & . & . \\
\hline
\end{tabular}


Tab. 5. - continued

\begin{tabular}{|c|c|c|c|c|c|c|}
\hline Succesive number & & 1 & 2 & 3 & 4 & 5 \\
\hline Number of relevé & & 12 & 14 & 9 & 21 & 10 \\
\hline Author & & DSV & CFP & $\mathrm{DZ}$ & GKL & $\mathrm{AC}$ \\
\hline$\underline{\text { Sign }}$ & & LS & $\mathrm{CG}$ & GB & $\mathrm{CP}$ & GP \\
\hline \multicolumn{7}{|l|}{ Artemisietea vulgaris } \\
\hline Erigeron annuиs & E1 & 8 & . & . & . & . \\
\hline Melilotus albus & E1 & 8 & . & . & . & . \\
\hline \multicolumn{7}{|l|}{ Other species } \\
\hline Festuca sp. & E1 & 8 & . & . & . & . \\
\hline Mentha sp. & E1 & 8 & . & . & . & . \\
\hline Robinia pseudacacia & E1 & 8 & . & . & . & . \\
\hline Phalaris arundinacea & E1 & . & . & 11 & . & . \\
\hline Salix cinerea & E1 & . & . & 11 & . & . \\
\hline Fragaria vesca & E1 & . & . & . & . & 80 \\
\hline Verbascum nigrum & E1 & . & . & . & . & 30 \\
\hline Geranium phaeum & E1 & . & . & . & . & 20 \\
\hline Juniperus communis & E1 & 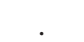 & 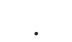 & . & . & 30 \\
\hline
\end{tabular}

1: Leontodonti-Seslerietum calcariae, this paper

2: Centaureo dichroanthae-Globularietum cordifoliae, Feoli Chiapella and Poldini 1993

3: Gentianello pilosae-Brometum erecti, Dakskobler and Završnik 2009

4: Cirsio-Peucedanetum cervariae, van Gils, Keysers and Launspach 1975

5: Geranio-Peucedanetum cervariae, Čarni 1998

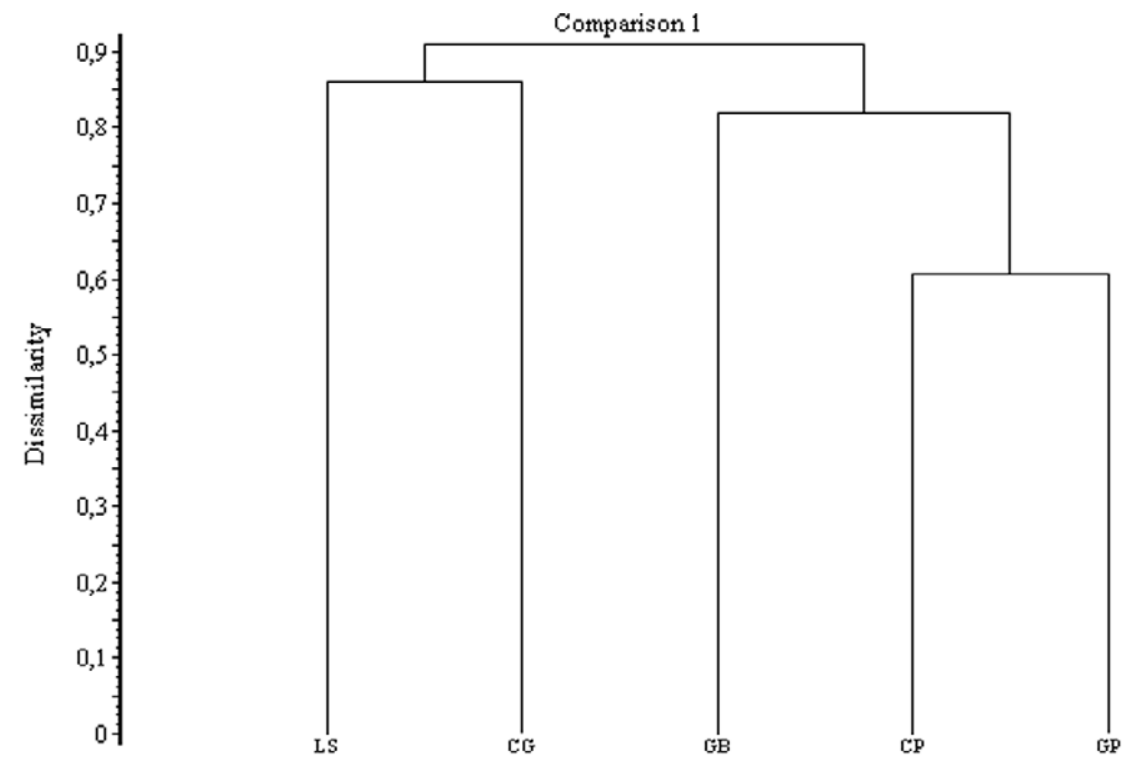

Fig. 10. Dendrogram of pioneer grassland and fringe vegetation (LS - Leontodonti-Seslerietum calcariae, CG - Centaureo dichroanthae-Globularietum cordifoliae, GB - Gentianello pilosae-Brometum erecti, CP - Cirsio pannonicae-Peucedanetum cervariae, GP - Geranio-Peucedanetum cervariae) - UPGMA, similarity ratio. 


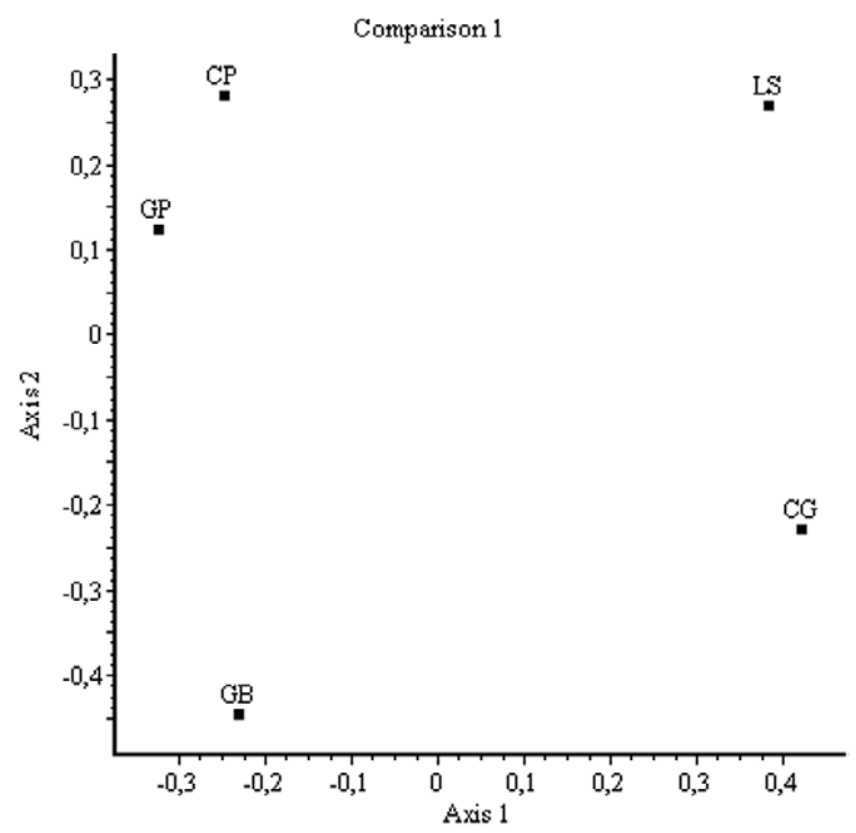

Fig. 11. Two-dimensional scatter-diagram of pioneer grassland and fringe vegetation (LS - Leontodonti-Seslerietum calcariae, CG - Centaureo dichroanthae-Globularietum cordifoliae, GB - Gentianello pilosae-Brometum erecti, $\mathrm{CP}$ - Cirsio pannonicae-Peucedanetum cervariae, GP - Geranio-Peucedanetum cervariae) - PCoA, similarity ratio.

Chiapella and Poldini 1993), Gentianello pilosae-Brometum erecti Dakskobler et Završnik 2009 (DAKSKOBLER and ZAVRŠNIK 2009), and two thermophilic forest edge communities - Cirsio pannonicae-Peucedanetum cervariae van Gils et al. 1975 (van GILs et al. 1975) and Geranio-Peucedanetum cervariae (KUHN 1937) T. Müller 1961 var. geogr. Knautia drymeia Čarni 1998 (ČARNI 1998) were ranged and compared using numerical methods. The results (Figs. 10, 11) clearly demonstrate that our stands are the most similar to the initial grassland on gravel sites of mountain rivers and streams in the foothills of the Southeastern Alps (association Centaureo dichroanthae-Globularietum cordifoliae) and are considerably different from thermophilic forest edge communities. Their floristic composition is also clearly different from riparian stands of the association Gentianello pilosae-Brometum erecti. This community is much more species-rich and grows on more developed soils, rendzinas. The stands of the association Leontodonti brumatii-Seslerietum calcariae are a successional stage in the afforestation of riparian rocks towards scrub and forest communities (Seslerio albicantis-Ostryetum, Ostryo-Fagetum). In addition to some meadow species they are mainly characterized by diagnostic species or rock crevices, screes and basophilic pine forests. Diagnostic species of the new association are Leontodon hispidus subsp. brumatii, Sesleria caerulea subsp. calcaria, Calamagrostis varia, Hieracium porrifolium, Aster bellidiastrum and Athamanta turbith. The holotypus of the new association is relevé No. 7 in table 2. The new association is classified as an explicitly fringe association (non-typical; with its species composition it is close also to the classes Elyno-Seslerietea, Thlapietea rotundifolii and Asplenietea trichomanis), into the Illyrian-pre-Alpine 
suballiance Centaurenion dichroanthae, into the alliance Saturejion subspicatae, order Scorzonero-Chrysopogonetalia and class Festuco-Brometea. It is subdivided into two subassociations. The differential species of the subassociation Leontodonti-Seslerietum chamaecytisetum purpureae subass nov. are Chamaecytisus purpureus, Carex humilis, Allium ericetorum, Centaurea scabiosa subsp. fritschii and Inula ensifolia. Its nomenclatural type is the holotypus of the association (relevé No. 7 in table 2); its sites are limestone riparian rocks. It is still flooded when the water level is high, but soil has already started accumulating in the runnels, which allows for a progressive development of the vegetation. The differential species of the subassociation Leontodonti-Seslerietum saxifragetum crustatae subass. nov. are Saxifraga crustata, Erica carnea, Laserpitium prutenicum, Campanula cespitosa, Petasites paradoxus and Stachys recta. The nomenclatural type, holotypus, is relevé No.13 in table 2. The sites of this subassociation are steep, gravelly, erosion slopes on platy limestone with addition of chert on the northeastern slopes of Bučenica, some ten metres above the Soča between Tolmin and Most na Soči. The species composition indicates the development from a scree community through stony grassland into a thermophilic forest (Ostryo-Fagetum). The first three relevés in table 2 could not be classified into the new association. The floristic composition of relevé No. 1 in table 2 (rocky eyot in the Idrijca at Slap ob Idrijci) shows some similarities with the stands of the association Triseto-Leontodontetum, while relevés 2 and 3 in the same table represent a chasmophytic community, for the time being synsystematically still undefined, at the road cut between Podsela and Doblar, dominated by Athamanta turbith, Campanula carnica and C. pyramidalis.

\section{Discussion}

The new localities of the southeastern-Alpine endemic Leontodon hispidus subsp. brumatii in the Sava valley (central Slovenia, the Black Sea river basin), in the gorge of the Sava between the village of Sava and Zidani Most, are rather surprising, because so far this taxon was known in Slovenia only in the Soča basin (on riparian rocks along the Soča, Nadiža, Učja, Idrija and Idrijca), which belongs the Adriatic Sea river basin, but not along other Alpine rivers. Nevertheless, the new localities are situated in the region where quite a few species with predominantly Alpine distribution grow (e.g. Rhododendron hirsutum, Paederota lutea, Pinguicula alpina, Rosa pendulina, Cerastium subtriflorum, Tephroseris pseudocrispa, Aster bellidiastrum, Campanula carnica, C. cespitosa, Trisetum argenteum, Arabis alpina subsp. alpina, Astrantia carniolica, Acinos alpinus, Aconitum degenii subsp. paniculatum, Tofieldia calyculata, Myrrhis odorata - the latter is mentioned by PETKOVŠEK 1939). The flora of the Sava valley also comprisessome species that most frequently occur in the Soča valley, but are usually much rarer in other landscape regions of Slovenia. Such species are for example Saxifraga petraea, Veratrum nigrum, Hemerocallis lilioasphodelus, Spiraea chamaedryfolia. There are also some similarities between the forest communities of valuable broad-leaved species (e.g. the probable occurrence of the stands of two associations described in the Soča valley, Veratro nigri-Fraxinetum and Saxifrago petraeae-Tilietum, also in the Sava valley). It is also likely that the taxon L. brumatii occurs on suitable sites elsewhere in the Sava basin (and other Slovenian Alpine rivers), but has been overlooked. Riparian sites are usually difficult to access and are therefore not the subject of a more detailed floristic inventory. The established differences in the communities 
of L. brumatii between the Soča and the Sava valleys do not rule out the possibility that the stands of the association Triseto-Leontodontetum that was described in the Sava valley occur also in the Soča valley (which is very likely, because the sites in the Soča valley comprise a higher number of this endemic than those in the Sava valley). Similarly, the occurrence of the stands of the association Leontodonti-Seslerietum described in the Soča valley, is likely also in the Sava valley (already demonstrated in one of the relevés). Both newly described associations include phytocoenoses that are usually distributed in smaller areas and are more or less long-term successional stages. These are by nature subject to the river dynamics and can disappear from one spot only to reappear in another. They are extremely exposed to human interventions of any kind. The endemic Leontodon brumatii and its communities are a characteristic of riparian flora and vegetation of some of Slovenian mountain rivers. According to current knowledge, the localities in the Sava valley are explicitly disjunct and the southeasternmost in the entire known distribution area, so they deserve our attention and protection. The envisaged interventions along the central Sava, i.e. construction of new hydroelectric power plants, would most probably lead to destruction of suitable habitats for Leontodon brumatii. With a higher water level between Zagorje and Hrastnik, this endemic is likely to disappear from the river's riparian zone, as has probably already happened downstream, before Zidani Most or Radeče and further south.

\section{Acknowledgements}

The research in the Sava valley was conducted in the framework of the research programme Gradients and Biodiversity: Flora, Fauna and Vegetation (grant no. P1-0236), funded by Slovenian Research Agency, and of the assignment Inventory of plant species in the middle Sava area (commissioned by the HSE d.o.o.). Boško Čušin, MSc, helped us in the field. Associate professor Andraž Čarni, PhD and Urban Šilc, PhD, helped us with literature sources. Iztok Sajko prepared figures 1 and 2 for print. Sincere thanks to two anonymous reviewers for their helpful remarks, instructions, corrections and improvements. English translation by Andreja Šalamon Verbič.

\section{References}

Aeschimann, D., Lauber, K. D. M., Moser, D. M.,Theurillat, J.-P., 2004: Flora alpina, 2: Gentianaceae-Orchidaceae. Haupt Verlag, Bern, Stuttgart, Wien.

Božıč, G., Vilhar, U., Urbančič, M., Kobal, M., Ferreira, A., Kraigher, H.,Grebenc, T., Sinjur, I., ŠTupar, B. Hrenko, M., Verlič, A., Jarni, K., Brus, R., Čarni, A., ŠIll, U., KoŠIr, P., MARINŠEK A., DAKSKOBler, I., 2008: Population genetic studies and sites investigation of autochthonous European black poplar (Populus nigra L.) along river basins and floodplain forest areas in Slovenia and guidelines for its conservation. Gozdarski inštitut Slovenije, Ljubljana.

Braun-Blanquet, J., 1964: Pflanzensoziologie. Grundzüge der Vegetationskunde, 3. Springer Verlag, Wien-New York.

Balátová-TuláčKová, E., Mucina, L., Ellmauer, T., WallnöFfer S., 1993: Phragmiti-Magnocaricetea. In: Grabherr, G., MucinA, L. (eds.), Die Pflanzengesellschaften 
Österreichs, 2. Natürliche waldfreie Vegetation. Gustav Fischer Verlag, Jena, Stuttgart, New York

BusER, S., 1990: Geological map of Slovenia 1: 500 000. Geodetski zavod Slovenije and Mladinska knjiga, Ljubljana.

Cegnar, T., 1998: Average annual, January and July air temperature between 1961 and 1990 (in Slovenian). In: Fridl, J., Kladnik, D., Orožen Adamič, M., Perko, D. (eds.), Geografski atlas Slovenije: država v prostoru in času, 101-103, DZS, Ljubljana.

ČARNI, A., 1998: La végétation des ourlets naturels dans la région préedinarique en Slovénie. Documents phytosociologiques N. S. 8, 119-138.

ČUšıN, B., 2001: A contribution to the Flora of the Breginjski kot (western Slovenia) (in Slovenian). Hladnikia (Ljubljana) 11, 5-16.

ČušIN, B., 2006: The flora of the Breginjski kot (in Slovenian). Založba ZRC, ZRC SAZU, Ljubljana.

ČUŠIN, B., DAKSKOBLER, I., 2001: Floristic novelities from the Soča valley (northwestern and western Slovenia) (in Slovenian). Razprave - Dissertationes 4. razreda SAZU (Ljubljana) 42-2, 5, 63-85.

DAKSKOBLER, I., 2005: Floristic novelities from the Soča valley and adjoining (adjacent) areas in western and northwestern Slovenia, 4 (in Slovenian). Hacquetia (Ljubljana) 4, $173-200$.

DAKSKOBLER, I., 2010: Development of vegetation on gravel sites of the Idrijca River in western Slovenia. Folia Biologica et Geologica (Ljubljana) 51, 5-90.

DAKSKOBLER, I., ZAVRŠNIK, K., 2009: Phytosociological and floristic analyses of riverine meadows at the village of Soča (the Julian Alps) and proposals for their protection (in Slovenian). Annales Series Historia Naturalis (Koper) 19, 63-82.

EHRENDORFER, F., HAMANN, U., 1965: Vorschläge zu einer floristischen Kartierung von Mitteleuropa. Berichte der Deutschen Botanischen Gesellschaft 78, 35-50.

Feoli Chiapella, L., Poldini, L., 1993: Prati e pascoli del Friuli (NE Italia) su substrati basici. Studia Geobotanica 13, 3-140.

HRIVINÁK, R., UjHÁZY, K., 2003: The stands with the Phalaroides arundinacea dominance in the Ipel' river catchment area (Slovakia and Hungari). Acta Botanica Hungarica 45, 297-314.

Hydrologicaldata Archive, 2008: Data archive for 2008. Agencija Republike Slovenije za okolje. Ljubljana (http://vode.arso.gov.si/hidarhiv; searched on 25 October 2011).

Jogan, N., Bačič, T., Frajman, B., Leskovar, I., Naglič, D., Podobnik, A., Rozman, B., StrgulC-KRAJŠEK S., TRČAK, B., 2001: Materials for the Atlas of Flora of Slovenia (in Slovenian). Center za kartografijo favne in flore, Miklavž na Dravskem polju.

MAAREL van der, E., 1979: Transformation of cover-abundance values in phytosociology and its effects on community similarity. Vegetatio 39, 2, 97-114.

MARTINČIČ, A., 2003: The list of mosses (Bryopsida) of Slovenia. Hacquetia (Ljubljana) 2, 91-166.

Martinčič, A., Wraber, T., Jogan, N., Podobnik, A., Turk, B., Vreš, B., Ravnik, V., Frajman, B., Strgulc Krajšek, S., TrČak, B., Bačič, T., Fischer, M. A., Eler, K., SuRINA, B., 2007: Small Flora of Slovenia. Identification key of vascular plants (in Slovenian). Tehniška založba Slovenije, Ljubljana. 
MAYER, E., 1952: Verzeichnis der Farn- und Blütenpflanzen des slowenischen Gebietes (in Slovenian). Academia scientiarum et artium Slovenica, 4: Historia naturalis et medicina. Opera 5, 1-427.

MAYeR, E., 1958: Zur Kenntnis der Flora der Westlichen Julischen Alpen. Razprave Dissertationes 4. razreda SAZU (Ljubljana) 4, 5-37.

MAYER, E., 1960: Endemische Blütenpflanzen der südöstlichen Kalkalpen, ihres Voralpenund illyrischen Übergangsgebietes (in Slovenian). Ad annum Horti botanici Labacensis solemnem, 25-45, Ljubljana.

Perko, D., Orožen AdAmič, M. (eds.), 1998: Slovenia. Landscapes and people (in Slovenian). Mladinska knjiga, Ljubljana.

PetKovŠEK, V., 1939: Mountain flowers in lowland (in Slovenian). Planinski vestnik (Ljubljana) 39, 65-71, 93-119.

PetrineC, V., 1999: Vegetationsmonographie von Šturmovci (NO Slowenien). Bachelor's thesis. Formal- und Naturwissenschaftliche Fakultät der Universität Wien.

PoDANI, J., 2001: SYN-TAX 2000. Computer Programs for Data Analysis in Ecology and Systematics. User's Manual, Budapest.

PoldinI, L., 1991: Atlante corologico delle piante vascolari nel Friuli-Venezia Giulia. Inventario floristico regionale. Regione Autonomo Friuli-Venezia Giulia et Università di Trieste, Udine.

PoldinI, L., 2002: Nuovo Atlante corologico delle piante vascolari nel Friuli Venezia Giulia. Regione Autonoma Friuli Venezia Giulia, Azienda Parchi e Foreste Regionali et Università degli Studi di Trieste, Dipartimento di Biologia, Udine.

PoldinI, L., 2009: La diversità vegetale del Carso fra Trieste e Gorizia. Lo stato dell' ambiente. Edizione Goliardiche, Trieste.

SelišKar, T., VReš, B., SelišKar A., 2003: FloVegSi 2.0. Fauna, Flora, Vegetation and Paleovegetation of Slovenia. Computer programme for arranging and analysis of biological data. Jovan Hadži Institute of Biology SRC SASA, Ljubljana.

Theurillat, J.-P., 2004: Pflanzensoziologisches System. In: Aeschimann, D., LAuber, K., D. M. Moser, D. M., Theurillat, J.-P. (eds.), Flora alpina 3: Register, 301-313. Haupt Verlag, Bern.

van Gils, H., Kaysers, E., Launspach, W., 1975: Saumgesellschaften im klimazonalen Bereich des Ostryo-Carpinion orientalis. Vegetatio 31,1, 47-64.

WraBer, M., 1969: Pflanzengeographische Stellung und Gliederung Sloweniens. Vegetatio $17,176-199$.

Wraber, T., 1998: Notulae ad nomenclaturum editionis Mala flora Slovenije anni 1999 spectantes. Hladnikia (Ljubljana) 10, 41-43.

Wraber, T., 2007: Cichoriaceae [Compositae subfam. Cichorioideae]. In: Martinčič, A. (ed.), Mala flora Slovenije, 687-716. Tehniška založba Slovenije, Ljubljana.

ZuPANČIČ, B., 1998: Average annual precipitations between 1961 and 1990 (in Slovenian). In: Fridl, J., Kladnik, D., OrožEn AdAmič, M., Perko, D. et al. (eds.), Geografski atlas Slovenije: država v prostoru in času, 99, DZS, Ljubljana. 